\title{
HESS J1943+213: An Extreme Blazar Shining through the Galactic Plane
}

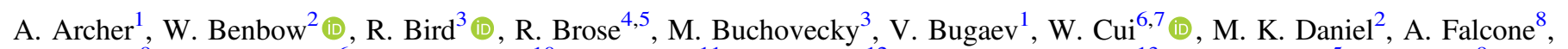
Q. Feng ${ }^{9}$ (i) , J. P. Finley ${ }^{6}$ (i) , A. Flinders ${ }^{10}$, L. Fortson ${ }^{11}$, A. Furniss ${ }^{12}$ (1) , G. H. Gillanders ${ }^{13}$ (1) , M. Hütten ${ }^{5}$, D. Hanna ${ }^{9}$ (1), O. Hervet ${ }^{14}$, J. Holder ${ }^{15}$, G. Hughes ${ }^{2}$, T. B. Humensky ${ }^{16}$, C. A. Johnson ${ }^{14}$ (D) P. Kaaret ${ }^{17}$ (D) , P. Kar ${ }^{10}$, N. Kelley-Hoskins ${ }^{5}$, D. Kieda ${ }^{10}$ (1), M. Krause ${ }^{5}$ (1) , F. Krennrich ${ }^{18}$, S. Kumar ${ }^{15}$, M. J. Lang ${ }^{13}$ (ㅁ), T. T. Y. Lin ${ }^{9}$, S. McArthur ${ }^{6}$, P. Moriarty ${ }^{13}$,

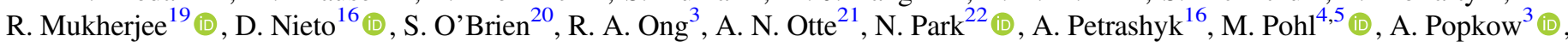
E. Pueschel ${ }^{5}$ (1) J. Quinn ${ }^{20}$, K. Ragan ${ }^{9}$, P. T. Reynolds ${ }^{23}$, G. T. Richards ${ }^{21}$ (1) , E. Roache ${ }^{2}$, C. Rulten ${ }^{11}$, I. Sadeh ${ }^{5}$, G. H. Sembroski ${ }^{6}$, K. Shahinyan ${ }^{11}$ (1) J. Tyler ${ }^{9}$, S. P. Wakely ${ }^{22}$, O. M. Weiner ${ }^{16}$, A. Weinstein ${ }^{18}$, R. M. Wells ${ }^{18}$, P. Wilcox ${ }^{17}$, A. Wilhelm ${ }^{4,5}$, D. A. Williams ${ }^{14}$ (1)

The VERITAS Collaboration,

W. F. Brisken ${ }^{24}$, and P. Pontrelli ${ }^{14}$

${ }^{1}$ Department of Physics, Washington University, St. Louis, MO 63130, USA

${ }^{2}$ Fred Lawrence Whipple Observatory, Harvard-Smithsonian Center for Astrophysics, Amado, AZ 85645, USA

${ }^{3}$ Department of Physics and Astronomy, University of California, Los Angeles, CA 90095, USA

${ }^{4}$ Institute of Physics and Astronomy, University of Potsdam, D-14476 Potsdam-Golm, Germany ${ }^{5}$ DESY, Platanenallee 6, D-15738 Zeuthen, Germany

${ }^{6}$ Department of Physics and Astronomy, Purdue University, West Lafayette, IN 47907, USA

${ }^{7}$ Department of Physics and Center for Astrophysics, Tsinghua University, Beijing 100084, People's Republic of China

${ }^{8}$ Department of Astronomy and Astrophysics, 525 Davey Lab, Pennsylvania State University, University Park, PA 16802, USA

${ }^{9}$ Physics Department, McGill University, Montreal, QC H3A 2T8, Canada

${ }^{10}$ Department of Physics and Astronomy, University of Utah, Salt Lake City, UT 84112, USA

${ }^{11}$ School of Physics and Astronomy, University of Minnesota, Minneapolis, MN 55455, USA; shahin@astro.umn.edu

${ }_{12}$ Department of Physics, California State University-East Bay, Hayward, CA 94542, USA

${ }^{13}$ School of Physics, National University of Ireland Galway, University Road, Galway, Ireland

${ }^{14}$ Santa Cruz Institute for Particle Physics and Department of Physics, University of California, Santa Cruz, CA 95064, USA; ohervet@ucsc.edu

${ }^{15}$ Department of Physics and Astronomy and the Bartol Research Institute, University of Delaware, Newark, DE 19716, USA

${ }^{16}$ Physics Department, Columbia University, New York, NY 10027, USA

${ }^{17}$ Department of Physics and Astronomy, University of Iowa, Van Allen Hall, Iowa City, IA 52242, USA

${ }^{18}$ Department of Physics and Astronomy, Iowa State University, Ames, IA 50011, USA

${ }^{19}$ Department of Physics and Astronomy, Barnard College, Columbia University, NY 10027, USA

${ }^{20}$ School of Physics, University College Dublin, Belfield, Dublin 4, Ireland

${ }^{21}$ School of Physics and Center for Relativistic Astrophysics, Georgia Institute of Technology, 837 State Street NW, Atlanta, GA 30332-0430, USA

${ }^{22}$ Enrico Fermi Institute, University of Chicago, Chicago, IL 60637, USA

${ }^{23}$ Department of Physical Sciences, Cork Institute of Technology, Bishopstown, Cork, Ireland

${ }^{24}$ Long Baseline Observatory, PO Box O, Socorro, NM 87801, USA

Received 2017 June 28; revised 2018 June 7; accepted 2018 June 8; published 2018 July 20

\begin{abstract}
HESS J1943+213 is a very high energy (VHE; $>100 \mathrm{GeV}$ ) $\gamma$-ray source in the direction of the Galactic plane. Studies exploring the classification of the source are converging toward its identification as an extreme synchrotron BL Lac object. Here we present $38 \mathrm{hr}$ of VERITAS observations of HESS J1943+213 taken over 2 yr. The source is detected with a significance of $\sim 20$ standard deviations, showing a remarkably stable flux and spectrum in VHE $\gamma$-rays. Multifrequency Very Long Baseline Array (VLBA) observations of the source confirm the extended, jetlike structure previously found in the $1.6 \mathrm{GHz}$ band with the European VLBI Network and detect this component in the 4.6 and $7.3 \mathrm{GHz}$ bands. The radio spectral indices of the core and the jet and the level of polarization derived from the VLBA observations are in a range typical for blazars. Data from VERITAS, Fermi-LAT, Swift-XRT, the FLWO 48" telescope, and archival infrared and hard X-ray observations are used to construct and model the spectral energy distribution (SED) of the source with a synchrotron self-Compton model. The well-measured $\gamma$-ray peak of the SED with VERITAS and Fermi-LAT provides constraining upper limits on the source redshift. Possible contribution of secondary $\gamma$-rays from ultra-high-energy cosmic-ray-initiated electromagnetic cascades to the $\gamma$-ray emission is explored, finding that only a segment of the VHE spectrum can be accommodated with this process. A variability search is performed across X-ray and $\gamma$-ray bands. No statistically significant flux or spectral variability is detected.
\end{abstract}

Key words: astroparticle physics - BL Lacertae objects: individual (HESS J1943+213, VER J1943+213) galaxies: active - galaxies: jets - galaxies: nuclei - gamma rays: galaxies

\section{Introduction}

Blazars are active galactic nuclei (AGNs) in which the axis of the relativistic jet is closely aligned with our line of sight (Urry \& Padovani 1995). The spectral energy distribution (SED) of blazars is characterized by a double-hump structure. In the simpler synchrotron self-Compton (SSC) models, the lower-energy hump is attributed to synchrotron emission from relativistic leptons, whereas the higher-energy hump is thought to be from inverse Compton upscattering of the synchrotron photons on the same population of relativistic leptons (Marscher 1980; Konigl 1981; 
Reynolds 1982). In more complicated scenarios, such as external Compton models, an external photon field, typically from the accretion disk or the dusty torus around the central black hole, is required to explain the higher-energy hump (e.g., Sikora et al. 1994). Alternatively, part or all of the $\gamma$-ray emission may be attributed to a hadronic origin, with proton synchrotron radiation and pion production constituting the two primary mechanisms (Mücke \& Protheroe 2001; Mücke et al. 2003). Variability is a common attribute of blazars, with variations in flux and spectrum detected in every observed frequency band and over a wide range of timescales (see Böttcher 2007).

Blazars come in two flavors: BL Lacertae objects (BL Lacs) and flat-spectrum radio quasars (FSRQs), with BL Lacs exhibiting lower-power jets and higher Doppler factors and FSRQs possessing high-powered jets and showing high Compton dominance (Stickel et al. 1991, 1993). Based on the location of the synchrotron peak, BL Lacs are classified into low, intermediate, and high synchrotron peak BL Lacs (LBLs, IBLs, and HBLs; respectively; Padovani \& Giommi 1995). HBLs are the most commonly detected blazars in VHE $\gamma$-rays, composing 47 of the 64 VHE-detected blazars. ${ }^{25}$ A subclass of HBLs has been proposed, known as extreme HBLs (EHBLs), identified by synchrotron emission peaks at energies above $1 \mathrm{keV}$ (Costamante et al. 2001).

Within the context of the blazar sequence (e.g., Fossati et al. 1998; Ghisellini \& Tavecchio 2008; Meyer et al. 2011; Giommi et al. 2012)—where the blazar jet luminosity is inversely related to the Doppler factor-EHBLs would be the least luminous and would have the highest Doppler boosting factors, making them one of the most efficient and extreme accelerators in the universe. However, with only a handful of blazars belonging to the EHBL subclass (including 1ES 0229 +200, 1ES 0347-121, RGB J0710+591, and 1ES 1101 -232), there is as yet no conclusive physical explanation for them.

EHBLs constitute a challenge for leptonic emission models that tend to only accommodate the observed SEDs of these objects with unusually hard particle populations (e.g., Tavecchio et al. 2010). Moreover, unlike other blazars, they do not appear to exhibit rapid variability, despite predictions of large flux variations on short timescales by leptonic models. The higher synchrotron peak frequency could potentially explain this as an observational effect by shifting the more variable emission produced by higher-energy particles into the hard $\mathrm{X}$-ray band. The less energetic particles producing steadier emission would then be responsible for the emission in the commonly observed infrared to soft X-ray bands.

The lack of rapid flux variability and the hard VHE spectra make EHBLs attractive candidates for hadronic emission models. Their observed properties can be explained by synchrotron emission from relativistic protons within the jet and by proton-initiated electromagnetic cascades (Cerruti et al. 2015). As such, the more distant EHBLs are also ideal candidates for a proposed $\gamma$-ray emission mechanism in which at least a component of the observed VHE emission originates from ultra-high-energy cosmic rays (UHECRs) that propagate an appreciable fraction of the distance between the blazar and Earth before producing electromagnetic cascades along the line of sight (see Ferrigno et al. 2004; Essey \& Kusenko 2010; Bonnoli et al. 2015). If either mechanism is confirmed, EHBLs

\footnotetext{
25 tevcat2.uchicago.edu
}

would become one of the most likely sources for the acceleration sites of UHECRs, directly addressing one of the oldest questions in high-energy astrophysics.

\subsection{HESS J1943+213: An Extreme HBL}

HESS $\mathrm{J} 1943+213$ is a VHE $\gamma$-ray point source discovered during the H.E.S.S. Galactic plane scan (Abramowski et al. 2011). Since the discovery publication, the identity of HESS J1943+213 has been a topic of debate, with most of the observations suggesting that the source is a blazar, but with alternative possibilities including a pulsar wind nebula (PWN) and a $\gamma$-ray binary.

Assuming that the source is a $\gamma$-ray binary, Abramowski et al. (2011) used the lack of detection of a massive (O- or Betype) companion star to estimate a distance limit of greater than $\sim 25 \mathrm{kpc}$. This distance places the binary well beyond the extent of the Galactic disk and implies an X-ray luminosity 100-1000 times higher than luminosities of known $\gamma$-ray binaries. Hence, Abramowski et al. (2011) disfavor the $\gamma$-ray binary scenario.

The point-like appearance in X-rays and the soft VHE spectrum, with a power-law index of $\Gamma=3.1 \pm 0.3$, motivated Abramowski et al. (2011) to argue against the PWN scenario. However, $1.6 \mathrm{GHz}$ observations of the HESS J1943+213 counterpart with the European VLBI Network (EVN) detected an extended source, with an FWHM angular size of 15.7 mas (with 3.5 mas being the expected size for a point source; Gabányi et al. 2013). Based on this measurement, the brightness temperature of the counterpart was estimated to be $7.7 \times 10^{7} \mathrm{~K}$ and was used to argue against the blazar scenario, as the expected brightness temperature of VHE-detected HBLs is in the $10^{9}-10^{10} \mathrm{~K}$ range. In addition, Gabányi et al. (2013) employed a $1^{\prime}$ feature observed in the $1.4 \mathrm{GHz}$ Very Large Array (VLA) C-array configuration image to support the PWN hypothesis, with the assertion that the angular size of the feature is consistent with a Crab-like PWN placed at a distance of $17 \mathrm{kpc}$. However, a pulsar search with the Arecibo telescope resulted in a nondetection and a claim of no pulsar at the HESS J1943+213 location at $\sim 70 \%$ confidence (Straal et al. 2016).

As reported by Abramowski et al. (2011), all observations were found to be consistent with the blazar scenario, however, including the point-like nature in both X-rays and VHE, the soft VHE spectral index, and an (unpublished) featureless IR spectrum. In addition, Tanaka et al. (2014) argued in favor of an EHBL by constructing an SED and by drawing comparisons to a known EHBL, 1ES 0347-121. The case for the extreme blazar has been bolstered further with Peter et al. (2014) observing the near-infrared ( $K$-band) counterpart of HESS $\mathrm{J} 1943+213$ and claiming a detection of an elliptical host galaxy.

Recently, Straal et al. (2016) obtained very long baseline interferometry (VLBI) observations in the 1.5 and $5 \mathrm{GHz}$ bands using the e-Multiple Element Remotely Linked Interferometer Network (e-MERLIN), showing that the source exhibits a flat spectrum between the two bands and claiming a detection of flux density variability in the $1.5 \mathrm{GHz}$ band when compared with the EVN observations of the source.

A strong argument for the blazar case was made with a reanalysis of the initial EVN data set and addition of new, higher-resolution observations in 2014 (Akiyama et al. 2016). Based on both EVN observations, the brightness temperature of the core is estimated to be well within the blazar range with 
$T_{\mathrm{B}}>1.8 \times 10^{9} \mathrm{~K}$ and $7.7 \times 10^{9} \mathrm{~K}$ for 2011 and 2014 observations, respectively. The claim for flux density variability was also made more robust through a consistent analysis of EVN data from the two epochs. In addition, the 2014 EVN observations revealed extended jet-like structure with brightness temperatures of the individual substructures of the extended emission typical of AGN jets.

The arguments presented above strongly suggest that HESS $\mathrm{J} 1943+213$ is a BL Lac object behind the Galactic plane. With a synchrotron peak located at $\sim 10 \mathrm{keV}$ and with no apparent cutoff, HESS J1943+213 is classified as an extreme synchrotron BL Lac object or an EHBL. In addition to the location of the synchrotron peak, HESS J1943+213 displays other attributes of EHBLs, including a very large X-ray-to-radio flux ratio, weak emission in the $\mathrm{GeV}$ band, and a lack of strong flux variability relative to other blazars.

There are only indirect limits on the distance of HESS J1943 +213 measured by Peter et al. (2014). Lower limits on the redshift come from the assumed size for the host galaxy and measurement of its extension in near-IR, while upper limits are derived by extrapolating the Fermi-LAT spectrum into the VHE regime and assuming that the deviations from the extrapolated spectrum are entirely due to absorption by the extragalactic background light (EBL). The redshift bounds found by Peter et al. (2014) are $0.03<z<0.45$.

In what follows, we detail results from observations of HESS J1943+213 with VERITAS, Fermi-LAT, Swift-XRT, and VLBA and further characterize the properties of the source as an EHBL. Section 2 presents new observations and results collected with VERITAS and VLBA, in addition to analyses of $8 \mathrm{yr}$ of Fermi-LAT data, recent Swift-XRT observations, and long-term optical observations with the Fred Lawrence Whipple Observatory (FLWO) 48" telescope. The results from the analysis of HESS J1943+213 multiwavelength data are interpreted and discussed in Section 3, including a derivation of improved and more robust limits on the source redshift (Section 3.1) based on the $\gamma$-ray spectra from Fermi-LAT and VERITAS. We perform a search for variability in X-ray and $\gamma$-ray observations of HESS J1943+213 (Section 3.2), construct and model the broadband SED of the source (Section 3.3), and explore UHECR line-of-sight $\gamma$-ray production as an alternative emission mechanism (Section 3.4). We conclude in Section 4.

\section{Multiwavelength Observations of HESS J1943+213 and Data Analysis}

\subsection{Strong Detection and Characterization of the Source with VERITAS}

The Very Energetic Radiation Telescope Array System (VERITAS) is an imaging atmospheric Cerenkov telescope array located at the Fred Lawrence Whipple Observatory in southern Arizona $\left(31^{\circ} 40^{\prime} \mathrm{N}, 110^{\circ} 57^{\prime} \mathrm{W}, 1.3 \mathrm{~km}\right.$ a.s.1.). VERITAS is composed of four $12 \mathrm{~m}$ telescopes, each equipped with a 499 photomultiplier tube camera providing a 3.5 field of view (Holder et al. 2006). The array can reliably reconstruct $\gamma$-rays with energies between $85 \mathrm{GeV}$ and $30 \mathrm{TeV}$ and has an angular resolution at $68 \%$ containment of $<0^{\circ} .1$ for a $1 \mathrm{TeV}$ photon. The energy resolution is $17 \%$ at $1 \mathrm{TeV}$, with a $10^{5} \mathrm{~m}^{2}$ peak effective area (Park \& the VERITAS Collaboration 2015).

VERITAS observations of HESS J1943+213 took place over $\sim 2.5 \mathrm{yr}$ and are broken up into two periods for spectral analysis:
(I) 2014 May 27 (MJD 56,804)-2014 July 02 (MJD 56,840), and (II) 2015 April 20 (MJD 57,132)-2015 November 09 (MJD $57,335)$. The total exposure time of these observations is $37.2 \mathrm{hr}$, amounting to a weather-cleaned live time of $30.9 \mathrm{hr}$. Observations from Period I focused on deep exposures of the source and constitute $24.2 \mathrm{hr}$ of weather-cleaned data, while the remaining $6.7 \mathrm{hr}$ in Period II aimed at probing the source for variability. The source elevation during the VERITAS observations was within the $63^{\circ}-80^{\circ}$ range, with the common low-energy threshold for this analysis determined to be $180 \mathrm{GeV}$.

The analysis of the VERITAS data is performed and crossevaluated for consistency with the two independent, standard VERITAS analysis packages (Cogan 2008; Daniel 2008). The images of Cerenkov light from particle showers are parameterized with the classical Hillas approach (Hillas 1985). Standard cuts optimized for average source strength ( 5\% Crab Nebula flux) and spectral index $(\Gamma \sim 2.7)$ are used for separating $\gamma$-ray and cosmic-ray events (see Acciari et al. 2008, for details). The background for $\gamma$-ray-like events is measured using the reflected regions method (Fomin et al. 1994). The source significance is calculated using the generalized version of Equation (17) from Li \& Ma (1983) derived by Klepser (2012).

A source centered at $\alpha=19^{\mathrm{h}} 43^{\mathrm{m}} 59^{\mathrm{s}} \pm 1^{\mathrm{s}}$ (stat) $\pm 2^{\mathrm{s}}$ (sys) and $\delta=21^{\circ} 19^{\prime} 05^{\prime \prime} \pm 11^{\prime \prime}{ }_{\text {(stat) }} \pm 25^{\prime \prime}{ }_{\text {(sys) }}$ is detected with an excess of 19.3 standard deviations $(\sigma)$, consistent with the catalog position of HESS J1943+213. The VERITAS detection is fit by a two-dimensional Gaussian function representing the VERITAS point-spread function. The fit $\chi^{2} / \mathrm{NDF}$ is $2069 /$ 1931, where NDF is the number of degrees of freedom. This corresponds to a fit probability of $1.5 \%$. The best-fit Gaussian width is 0.05 , smaller than the angular resolution of VERITAS. Thus, there is no evidence for source extension. The VERITAS source name is VER J1943+213.

The VERITAS differential energy spectra of HESS J1943 +213 are constructed separately for each period specified above to look for variations in the source spectrum. The details of the observations and the spectral analysis for each period and for the combined dataset are provided in Table 1. The spectra from the two periods agree with each other within the statistical uncertainties, indicating no significant detection of spectral variability and justifying the use of a combined, time-averaged spectrum from the entire data set for the SED modeling detailed in Section 3.3. The combined VERITAS spectrum of HESS $\mathrm{J} 1943+213$ is presented in Figure 1 . The spectrum is fit well by a power-law function, $\frac{d N}{d E}=N_{0}\left(E / E_{0}\right)^{-\Gamma}$, with a spectral index $\Gamma=2.81 \pm 0.12_{\text {(stat }_{-0.34}}^{+0.14}$ in the $180 \mathrm{GeV}-2 \mathrm{TeV}$ energy range. Abramowski et al. (2011) reported a spectral index of $3.1 \pm 0.3_{\text {(stat) }} \pm 0.2_{\text {(sys) }}$ for HESS J1943+213 above $470 \mathrm{GeV}$. In the same energy range, the VERITAS best-fit spectral index is $2.85 \pm 0.32_{\text {(stat) }_{-0.34}^{+0.14} \text { (sys) }}$, consistent with the H.E.S.S. result.

There is no evidence for flux variability between Periods I and II and on weekly timescales as illustrated by the VERITAS light curve of HESS J1943+213 in Figure 2. The average flux

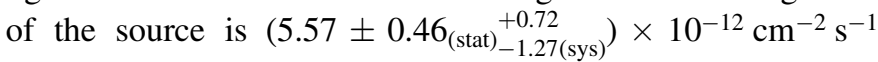
above $230 \mathrm{GeV}$. A constant line is fit to the full weekly binned light curve with $\chi^{2} / \mathrm{NDF}=15.6 / 12$, corresponding to a $p$-value of 0.21 for a constant flux. The higher-cadence Period I observations were investigated for variability separately on both daily and weekly timescales. No significant variability was found in that data set, with $\chi^{2} / \mathrm{NDF}=31.34 / 20$ for a 


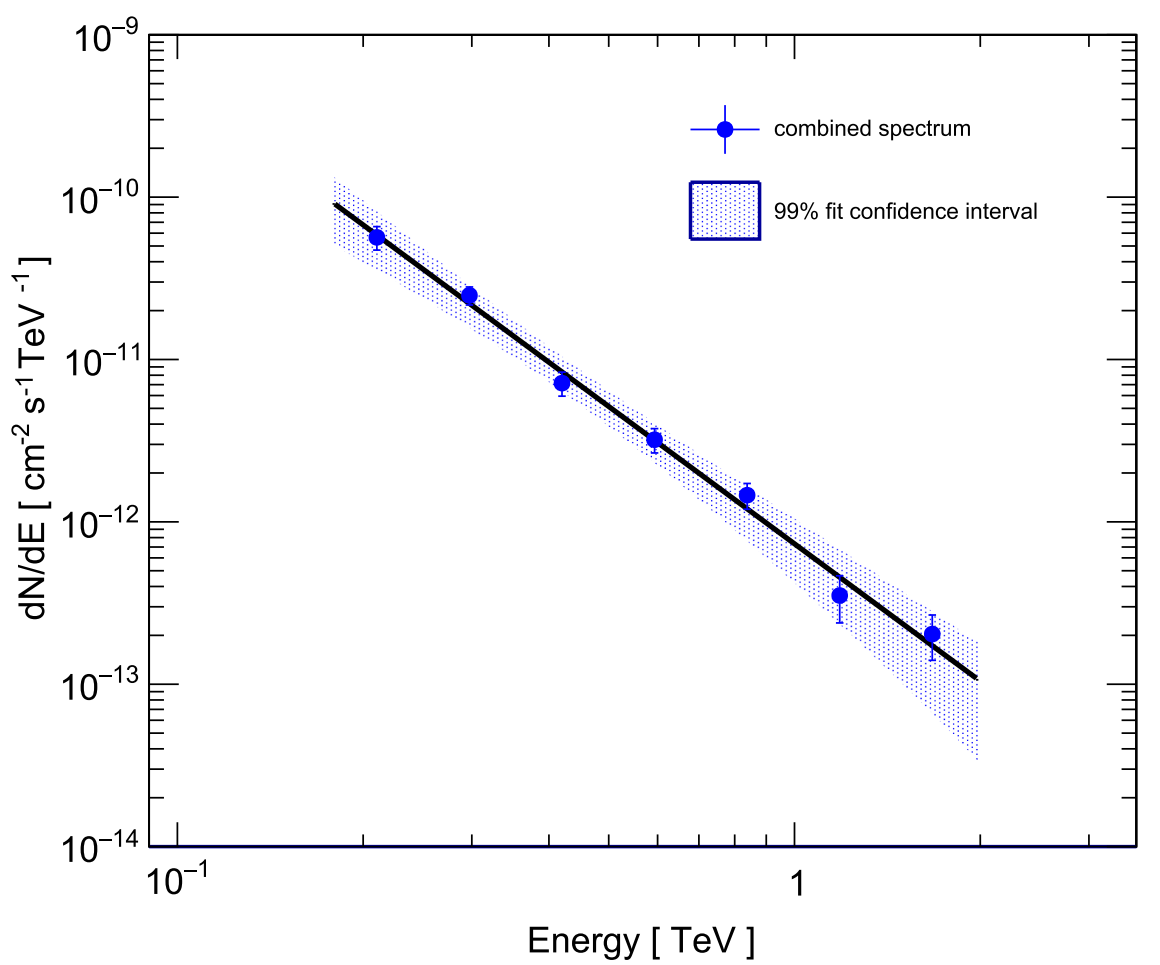

Figure 1. Time-averaged VERITAS spectrum of HESS J1943+213, combining data from 2014 and 2015 observations. The band shows the $99 \%$ confidence interval of a power-law fit to the spectrum.

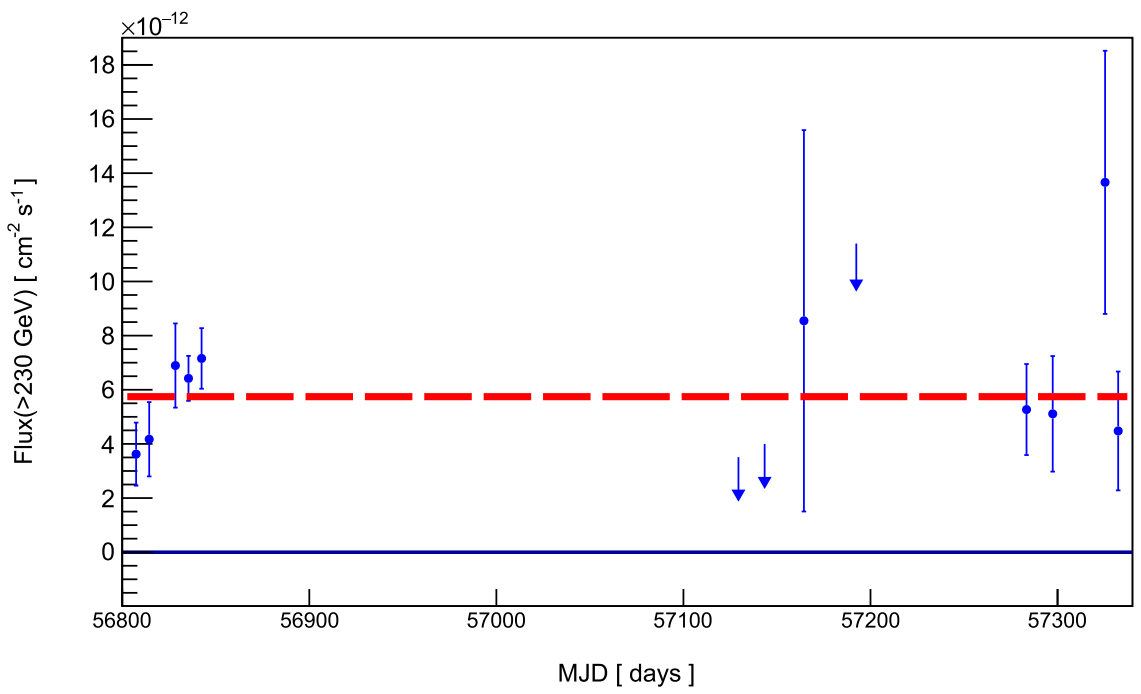

Figure 2. VERITAS light curve of HESS J1943+213 above $230 \mathrm{GeV}$. The red dashed line is a fit of a constant to the data. The $68 \%$ upper limits are derived for time bins in which the source flux is consistent with zero.

constant fit to a daily light curve, corresponding to a $p$-value of 0.05 for a constant flux (Shahinyan \& the VERITAS Collaboration 2015).

H.E.S.S. reported a source flux of $\left(1.3 \pm 0.2_{\text {(stat) }} \pm 0.3_{(\text {sys })}\right) \times$ $10^{-12} \mathrm{~cm}^{-2} \mathrm{~s}^{-1}$ above $470 \mathrm{GeV}$ (Abramowski et al. 2011) from observations taken between 2005 and 2008. This is consistent with the VERITAS flux above $470 \mathrm{GeV}$ of $(1.47 \pm$

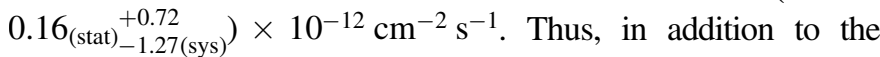
remarkable stability of the source flux over $2 \mathrm{yr}$ of VERITAS observations, there is also good agreement between fluxes from observations more than $6 \mathrm{yr}$ apart from two different VHE $\gamma$-ray instruments.

\subsection{Improved Detection and Spectral Analysis with an $8 y r$ Fermi-LAT Data Set}

The Large Area Telescope (LAT) on board the Fermi Gamma-ray Space Telescope (Fermi-LAT) is a pair-conversion $\gamma$-ray instrument sensitive to high-energy (HE) $\gamma$-rays with energies between $20 \mathrm{MeV}$ and $300 \mathrm{GeV}$. The LAT has a field of view that covers $\sim 20 \%$ of the sky at any given time in survey mode, providing 30 minutes of live time on each point in the sky every two orbits $(\sim 3 \mathrm{hr})$.

A source associated with HESS J1943+213 is included in the Second Catalog of Hard Fermi-LAT Sources (2FHL; Ackermann et al. 2016) within the $50 \mathrm{GeV}-2 \mathrm{TeV}$ energy 
Table 1

Summary of VERITAS Observations

\begin{tabular}{|c|c|c|c|c|c|c|c|c|}
\hline Period & $\begin{array}{l}\text { Exposure } \\
\text { (hr) }\end{array}$ & On Counts & Off Counts & $\alpha^{\mathrm{a}}$ & $\sigma$ & $\begin{array}{l}\text { Flux }(>180 \mathrm{GeV}) \\
\quad\left(\mathrm{cm}^{-2} \mathrm{~s}^{-1}\right)\end{array}$ & $\Gamma$ & $\chi^{2} / \mathrm{NDF}$ \\
\hline $\bar{I}$ & 24.2 & 713 & 7684 & 0.043 & 17.9 & $(8.61 \pm 0.78) \times 10^{-12}$ & $2.76 \pm 0.12$ & $3.5 / 6$ \\
\hline II & 6.7 & 164 & 2087 & 0.042 & 7.2 & $(8.55 \pm 1.88) \times 10^{-12}$ & $3.12 \pm 0.38$ & $4.4 / 4$ \\
\hline Combined & 30.9 & 877 & 9771 & 0.042 & 19.3 & $(8.61 \pm 0.67) \times 10^{-12}$ & $2.81 \pm 0.12$ & $3.8 / 5$ \\
\hline
\end{tabular}

Note.

${ }^{\mathrm{a}}$ Off region-source region normalization.

range, with a TS $=39.6$ and a power-law spectral index of $2.73 \pm 0.66$. In addition, the source is included in the preliminary Third Catalog of Hard Fermi-LAT Sources (3FHL; The Fermi-LAT Collaboration 2017) in the $10 \mathrm{GeV}-$ $2 \mathrm{TeV}$ range, with a $\mathrm{TS}=127.7$ and a spectral index of $1.45 \pm 0.29$. HESS J1943+213 has been previously detected at $5.1 \sigma$ significance through an analysis of $5 \mathrm{yr}$ of Fermi-LAT data in the $1-100 \mathrm{GeV}$ energy range, producing a spectrum well fit by a power law with normalization of $\left(3.0 \pm 0.8_{\text {(stat) }} \pm\right.$ $\left.0.6_{(\text {sys })}\right) \times 10^{-15} \mathrm{~cm}^{2} \mathrm{~s}^{-1} \mathrm{MeV}^{-1}$ at $15.1 \mathrm{GeV}$ and spectral index $\Gamma=1.59 \pm 0.19_{\text {(stat) }} \pm 0.13_{\text {(sys) }}$ (Peter et al. 2014).

In this work, the Fermi Science Tools ${ }^{26}$ version v10r0p5 and the P8R2_SOURCE_V6 instrument response functions are used for analyzing Fermi-LAT observations, with the assistance of Fermipy (Wood et al. 2017) — a python package with a high-level interface for Fermi-LAT analysis. Eight years of PASS8 Fermi-LAT data are selected for the analysis between 2008 August 04 and 2016 August 04. The region of interest (RoI) is defined within a $10^{\circ}$ radius of the catalog position of HESS J1943+213 $\left(\alpha: 19^{\mathrm{h}} 43^{\mathrm{m}} 55^{\mathrm{s}} ; \delta: 21^{\circ} 18^{\prime} 8^{\prime \prime}\right)$. SOURCE class events with energies in the $3-300 \mathrm{GeV}$ range are selected. The $3 \mathrm{GeV}$ lower bound on the energy is chosen to decrease the contribution from the Galactic diffuse background. In addition, only events with zenith angles $<100^{\circ}$ and rocking angles $<52^{\circ}$ are included to avoid contamination from the Earth limb.

A model for the RoI is constructed by including all FermiLAT Third Source Catalog (3FGL; Acero et al. 2015) sources within $15^{\circ}$ radius of the source position and models for emission from the Galactic diffuse ( $\left.g l l \_i e m \_v 06 . f i t s\right)$ and the isotropic (iso_P8R2_SOURCE_V6_v06.txt) backgrounds. In addition, a point source with a power-law spectrum is added at the catalog position of HESS J1943+213. A binned likelihood analysis is performed to find the optimal model for the RoI and extract the best-fit source parameters. The parameters of weak sources with test statistic (TS) $<16$ and all sources located more than $7^{\circ}$ away from the center of the RoI are frozen during this procedure.

A source at the position of HESS J1943+213 is detected with a TS value of 147.5 corresponding to a significance of $\sim 12 \sigma$. The source is modeled as a power-law function with an index of $1.67 \pm 0.11_{\text {(stat) }}$ and a flux of $\left(2.71 \pm 0.43_{\text {(stat) }}\right) \times 10^{-10} \mathrm{~cm}^{-2} \mathrm{~s}^{-1}$ above $3 \mathrm{GeV}$. Spectral points are generated by repeating the Fermi-LAT analysis with events selected within the energy range of each spectral bin. In addition, different spectral shapes are explored for the HESS J1943+213 detection showing no statistically significant preference for a curved model over a power law.

\footnotetext{
${ }^{26}$ https://fermi.gsfc.nasa.gov/ssc/data/analysis
}

\subsection{Swift-XRT Observations Contemporaneous with VERITAS}

X-ray observations of HESS J1943+213 were obtained with the Swift-XRT instrument on 2014 June 17, 19, and 21, contemporaneous with VERITAS observations. The XRT data analyzed here were collected in the photon counting mode, amounting to a total of 48.2 minutes of exposure time.

The XRT data analysis is performed with the standard XRTDAS v3.0.0 tools included in the HEASoft package Version 6.15.1, while Xspec (Arnaud 1996) v12.8.1g is used for the spectral analysis.

XRT spectra are constructed by unfolding the counts spectra with instrument response functions included in CALDB 1.0.2 and by assuming an absorbed power-law functional form for the intrinsic spectrum: $\frac{d N}{d E}=N_{0}\left(E / E_{0}\right)^{-\Gamma} e^{-N_{\mathrm{H}} \sigma(E)}$, where $\sigma(E)$ is the photoelectric cross section and $N_{\mathrm{H}}$ is the H I column density. Spectra were first fit using absorbed power-law functions with the $N_{\mathrm{H}}$ parameter left free to search for excess over the Galactic $N_{\mathrm{H}}$ value obtained from the Leiden/Argentine/Bonn (LAB) survey, $N_{\mathrm{H}} \sim 0.82 \times 10^{22} \mathrm{~cm}^{-2}$ (Kalberla et al. 2005). The fitted value of $N_{\mathrm{H}}$ is consistent with the value from the LAB survey for the observation on 2014 June $17\left(N_{\mathrm{H}}=(0.87 \pm 0.48) \times 10^{22} \mathrm{~cm}^{-2}\right)$ and slightly exceeds the LAB survey value for observations on 2014 June $19\left(N_{\mathrm{H}}=(1.48 \pm 0.54) \times 10^{22} \mathrm{~cm}^{-2}\right)$ and 2014 June $21\left(N_{\mathrm{H}}=(1.21 \pm 0.35) \times 10^{22} \mathrm{~cm}^{-2}\right)$. The high-precision SuzakuXIS and HXD/PIN spectrum from Tanaka et al. (2014) measured $N_{\mathrm{H}}=(1.38 \pm 0.03) \times 10^{22} \mathrm{~cm}^{-2}$, also in excess of the LAB survey value.

The spectrum from each observation is shown in Figure 3. Spectra are again constructed assuming absorbed power-law functional forms; however, the $N_{\mathrm{H}}$ parameter is kept fixed to the value from Tanaka et al. (2014). The results from the spectral fits are included in Table 2 . The uncertainties represent the $68 \%$ confidence intervals for the respective quantities. No significant spectral variability is detected between observations. Moreover, the results are comparable to measurements from 2006 October 10 observations of HESS J1943+213 with Swift-XRT, which reported a spectral index of $2.04 \pm 0.12$ and a flux of $(1.83 \pm 0.04) \times 10^{-11} \mathrm{erg} \mathrm{cm}^{-2} \mathrm{~s}^{-1}$ (Malizia et al. 2007; Landi et al. 2009).

\subsection{Long-term Optical Observations with FLWO 48"}

The FLWO 48" (1.2 m) telescope is located on Mt. Hopkins in southern Arizona. As part of a long-term, multiblazar observing program, optical photometry of HESS J1943+213 was obtained between 2013 September 27 and 2017 March 14 in Sloan Digital Sky Survey (SDSS) $r^{\prime}$ filter and between 2015 March 25 and 2017 March 14 in Harris $V$ and SDSS $i^{\prime}$ filters. The data reduction was performed using standard IDL tools. The magnitude zero-point was determined for each image by 


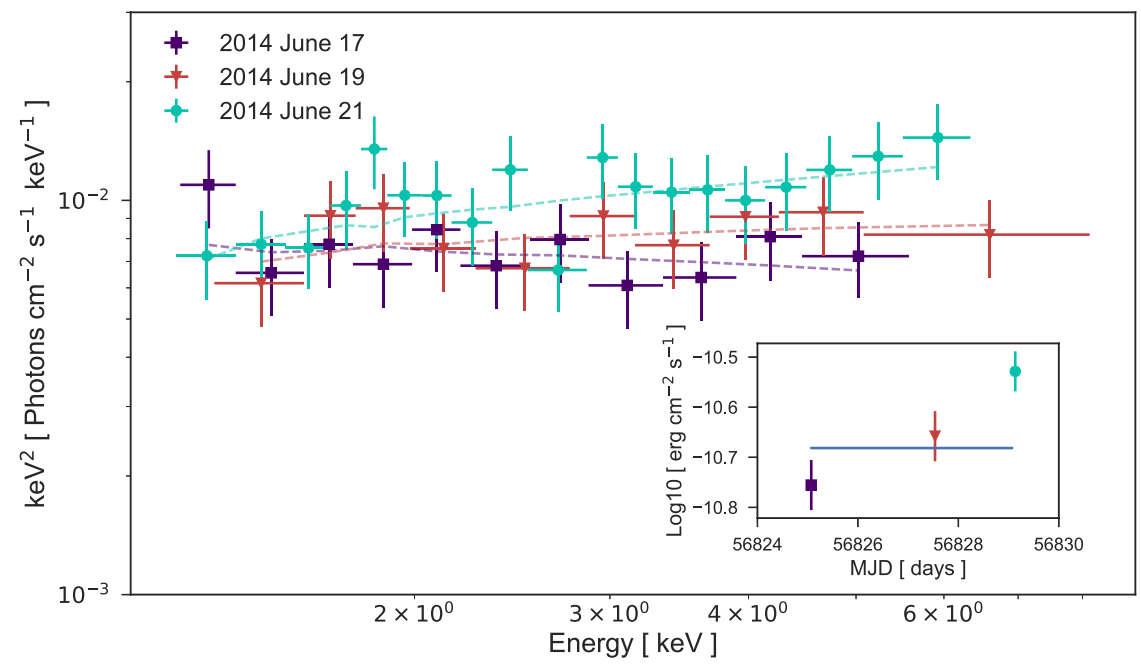

Figure 3. Spectra of HESS J1943+213 with Swift-XRT for the three observations taken in 2014. The dashed lines show the absorbed power-law models used for unfolding the spectra. The inset shows the $2-10 \mathrm{keV}$ fluxes for the three observations.

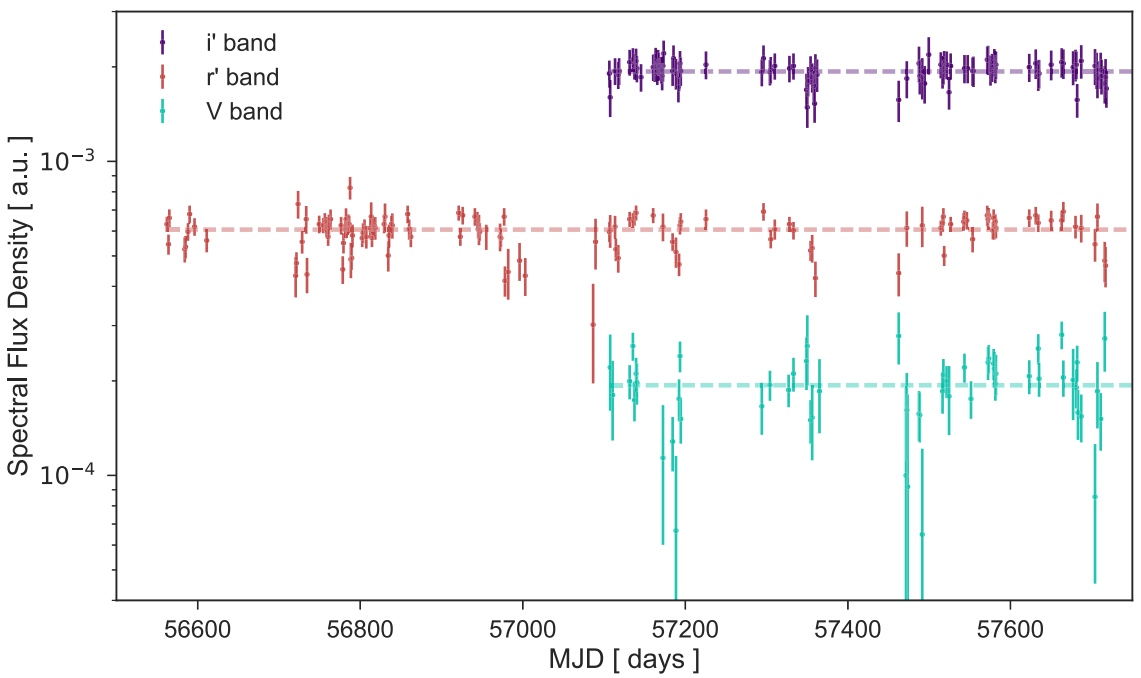

Figure 4. Light curves from FLWO 48" observations with Harris $V$ (teal), SDSS $r^{\prime}$ (red), and SDSS $i^{\prime}$ (purple) filters. The spectral flux densities are given in arbitrary units (a.u.). The dashed lines show the average flux for each of the light curves.

Table 2

Summary of Swift-XRT Observations

\begin{tabular}{lcccr}
\hline \hline Date & Observation ID & $\begin{array}{c}\text { Exposure } \\
(\mathrm{s})\end{array}$ & $\begin{array}{c}\log 10[\text { Flux] }(2-10 \mathrm{keV}) \\
\log 10\left(\mathrm{erg} \mathrm{cm}^{-2} \mathrm{~s}^{-1}\right)\end{array}$ & $\begin{array}{c}\text { Index } \\
\chi^{2} / \mathrm{NDF}\end{array}$ \\
\hline 2014 Jun 17 & 00033319001 & 967 & $-10.75 \pm 0.05$ & $2.16 \pm 0.18$ \\
2014 Jun 19 & 00033319002 & 769 & $-10.66 \pm 0.05$ & $1.92 \pm 0.16$ \\
2014 Jun 21 & 00033319003 & 1156 & $-10.53 \pm 0.04$ & $1.77 \pm 0.13$ \\
\hline
\end{tabular}

comparison to cataloged stars in order to derive the magnitude for HESS J1943+213 and a reference star located in the same field of view and only a few arcseconds from the source. The observations were not corrected for Galactic extinction, since extinction estimates at low Galactic latitudes are highly unreliable. Deviations of the reference star magnitude from the mean are used to reject observations from nights with poor weather. Magnitudes are converted to spectral flux densities assuming an $\mathrm{AB}$ magnitude system. The resulting light curves in the FLWO $48^{\prime \prime}$ Harris $V$ and SDSS $r^{\prime}$ and $i^{\prime}$ filters for HESS $\mathrm{J} 1943+213$ are presented in Figure 4. For brighter, nonvariable objects, such as the reference star used in this analysis, the distribution of measured magnitudes is consistent with the calculated statistical errors. We find, however, that for fainter objects with magnitude similar to HESS J1943+213, there is scatter in the measurements exceeding the calculated errors, indicating a dominant source of systematic error that remains to be identified. Hence, the FLWO $48^{\prime \prime}$ data are not used for a variability search. Average fluxes are derived for each band and included in the source SED in Section 3.3.

\subsection{Multifrequency VLBA Observations in 2015 and 2016}

The radio counterpart of HESS J1943+213 was observed by the authors using the VLBA. Observations took place over two 
epochs on 2015 August 11 (Project ID: BS246) and on 2016 August 08 (Project ID: BS253).

The 2015 observations (epoch I) were part of a request to follow up on the initial EVN detection and characterization of the source (Gabányi et al. 2013) by using four VLBA frequency bands $(1.6,4.3,7.6$, and $15 \mathrm{GHz})$. The epoch II observations taken in 2016 aimed to obtain deeper exposures of the source in $\mathrm{C}$ band (split into 4.3 and $7.6 \mathrm{GHz}$ bands) in order to characterize extended structures and measure polarization. The observations were targeted at the position reported from the EVN detection: $\alpha=19^{\mathrm{h}} 43^{\mathrm{m}} 56^{\mathrm{s}} 2372, \delta=21^{\circ} 18^{\prime} 23^{\prime \prime} 402$. All 10 VLBA antennae participated in both sets of observations. The total length of the 2015 observations was $4 \mathrm{hr}$, which included exposures on a phase calibrator source, J1946+2300, and a bandpass calibrator source, 3C 345. The 2016 observations totaled $8 \mathrm{hr}$ and included exposures on the same phase calibrator and bandpass calibrator sources, as well as an astrometric check source, J1935+2031, and a polarized calibrator, 3C 380.

The NRAO Astronomical Image Processing System ${ }^{27}$ (AIPS; van Moorsel et al. 1996) is used to reduce and calibrate the VLBA data for HESS J1943+213. Images from the 2015 observations were produced for each band and are displayed in Figure 5. There is clear evidence for extended, jet-like emission in the 1.6, 4.3, and 7.6 GHz images. This is the first detection of the extended milliarcsecond-scale structure from the HESS $\mathrm{J} 1943+213$ counterpart in 4.3 and $7.6 \mathrm{GHz}$ bands, allowing multifrequency exploration of its properties in VLBI. A similar core-jet structure has been previously detected with deep 1.6 GHz band observations with EVN (Akiyama et al. 2016). Images from the 2016 VLBA observations in 4.3 and $7.6 \mathrm{GHz}$ bands shown in Figure 6 reveal the source structure in more detail and provide fractional polarization measurements. The $1 \%-3 \%$ polarization of the core in both bands detected for the source is consistent with polarization levels seen in other $\gamma$-ray blazars (Linford et al. 2012). Quantitative results from the analysis of the two epochs of VLBA observations are provided in Table 3.

The core brightness temperature $\left(T_{\mathrm{B}}\right)$ of the HESS J1943 +213 counterpart is estimated using images from all bands except for the $15 \mathrm{GHz}$ band from 2015 observations, where the sensitivity was too low for phase and amplitude selfcalibration. Lower limits to $T_{\mathrm{B}}$ are derived owing to a partially resolved core and the possibility of interstellar scattering, resulting in $T_{\mathrm{B}}>1.2 \times 10^{9} \mathrm{~K}$ in the most conservative case, measured with the $1.6 \mathrm{GHz}$ image from the 2015 data set. The brightness temperature values are well within the range for blazars. We do not confirm the significantly lower brightness temperature measurement of $T_{\mathrm{B}}=7.7 \times 10^{7} \mathrm{~K}$, which was based on the EVN 1.6 GHz image (Gabányi et al. 2013) and has subsequently been reanalyzed showing higher brightness temperature, $T_{\mathrm{B}}>1.8 \times 10^{9} \mathrm{~K}$ (Akiyama et al. 2016).

Using the 2015 observations and assuming a power-law function of the form $S \propto \nu^{-\alpha}$, where $S$ is the spectral flux density, a spectral index $\alpha_{\text {core }}=0.3 \pm 0.06$ is measured for the HESS J1943+213 core, determined from all four bands, and an index $\alpha_{\text {jet }}=1.1 \pm 0.4$ for the extended emission is calculated over 9.3 mas $^{2}$ based on the 4.3 and $7.6 \mathrm{GHz}$ images. With the deeper 2016 data set, we construct a spectral index map and present it in Figure 7. In order to construct the map,

\footnotetext{
27 http://www.aips.nrao.edu
}

the $7.6 \mathrm{GHz}$ image is convolved with a larger beam size to match the resolution of the $4.3 \mathrm{GHz}$ image. There is a visible discrepancy between $\alpha_{\text {core }}=0.3 \pm 0.06$ determined from epoch I observations using the 1.6, 4.3, 7.6, and $15 \mathrm{GHz}$ images and the values shown in the spectral index map that are near $\alpha=-0.3$ at the center of the core. This is largely a result of differing resolutions for the four bands involved in the spectral index calculation for epoch I data. The epoch II spectral index map, which uses images with matched resolutions, is more robust and does not suffer from this issue.

Measurements of the spectral indices of the core and the extended structures from both epochs are consistent with reported values for blazar cores and jets from the MOJAVE sample (Hovatta et al. 2014). The only available radio spectral index measurements of the core of this source come from e-MERLIN observations, which find an index of $0.03 \pm 0.03$ (Straal et al. 2016). The e-MERLIN observations do not resolve the source, however, and the core spectral index calculation using these observations is affected by emission from extended structures.

Comparisons between EVN and VLBA $1.6 \mathrm{GHz}$ results show apparent changes in the core flux density. Gabányi et al. (2013) measured $31 \pm 3 \mathrm{mJy}$ for the source flux density with EVN, while the flux density measurement from our VLBA image is $23.6 \pm 0.2 \mathrm{mJy}$. A similar change in the source flux density was reported by Straal et al. (2016) between the 2011 EVN $1.6 \mathrm{GHz}$ result and a lower-resolution e-MERLIN $1.5 \mathrm{GHz}$ detection in 2013 of the source with $22.2 \pm 0.7 \mathrm{mJy}$, leading to the first variability claim for HESS $\mathrm{J} 1943+213$ or its counterparts in any band. As the core is not fully resolved in $1.6 \mathrm{GHz}$, however, and different configurations were used for the VLBA, EVN, and e-MERLIN observations, the claims for variability are not definitive.

e-MERLIN observations in the $5 \mathrm{GHz}$ band were also obtained by Straal et al. (2016), resulting in a $22.4 \pm 0.3 \mathrm{mJy}$ flux density, which is significantly higher than the VLBA $4.3 \mathrm{GHz}$ measurements of $\sim 16.2 \mathrm{mJy}$ in 2015 and $17.1 \mathrm{mJy}$ in 2016. The latter discrepancy could be explained by a change in the source flux density, but more likely by differences in $u v$ coverage between VLBA and e-MERLIN observations and the inclusion of the jet feature in the core flux density measurement with e-MERLIN.

Despite the strong arguments for classifying HESS J1943 +213 as an EHBL, a measurement of the proper motion can be a definitive discriminator between Galactic and extragalactic origin for the source. We attempt two sets of proper-motion measurements of the HESS J1943+213 VLBI counterpart. In the first case, we compare the position measurement from our pure phase-referenced (no self-calibration) VLBA $15 \mathrm{GHz}$ image from 2015 with the position reported from the Gabányi et al. (2013) EVN detection and find a change in position of 1.1 mas. This is consistent with zero, given the $\sim 2.5$ mas uncertainty in the position measurements, which is largely dominated by the uncertainty in the EVN position. Using this uncertainty and the $\sim 4.3 \mathrm{yr}$ time difference between the two observations, an upper limit of $47 \mathrm{~km} \mathrm{~s}^{-1}$ is calculated for the transverse velocity of the source at $17 \mathrm{kpc}$ - the assumed distance if the source is a Galactic PWN (Abramowski et al. 2011; Gabányi et al. 2013).

In the second case, we compare the positions from our 2015 and 2016 VLBA observations using the phase-referenced 4.3 GHz images almost exactly a year apart. The 2015 VLBA 

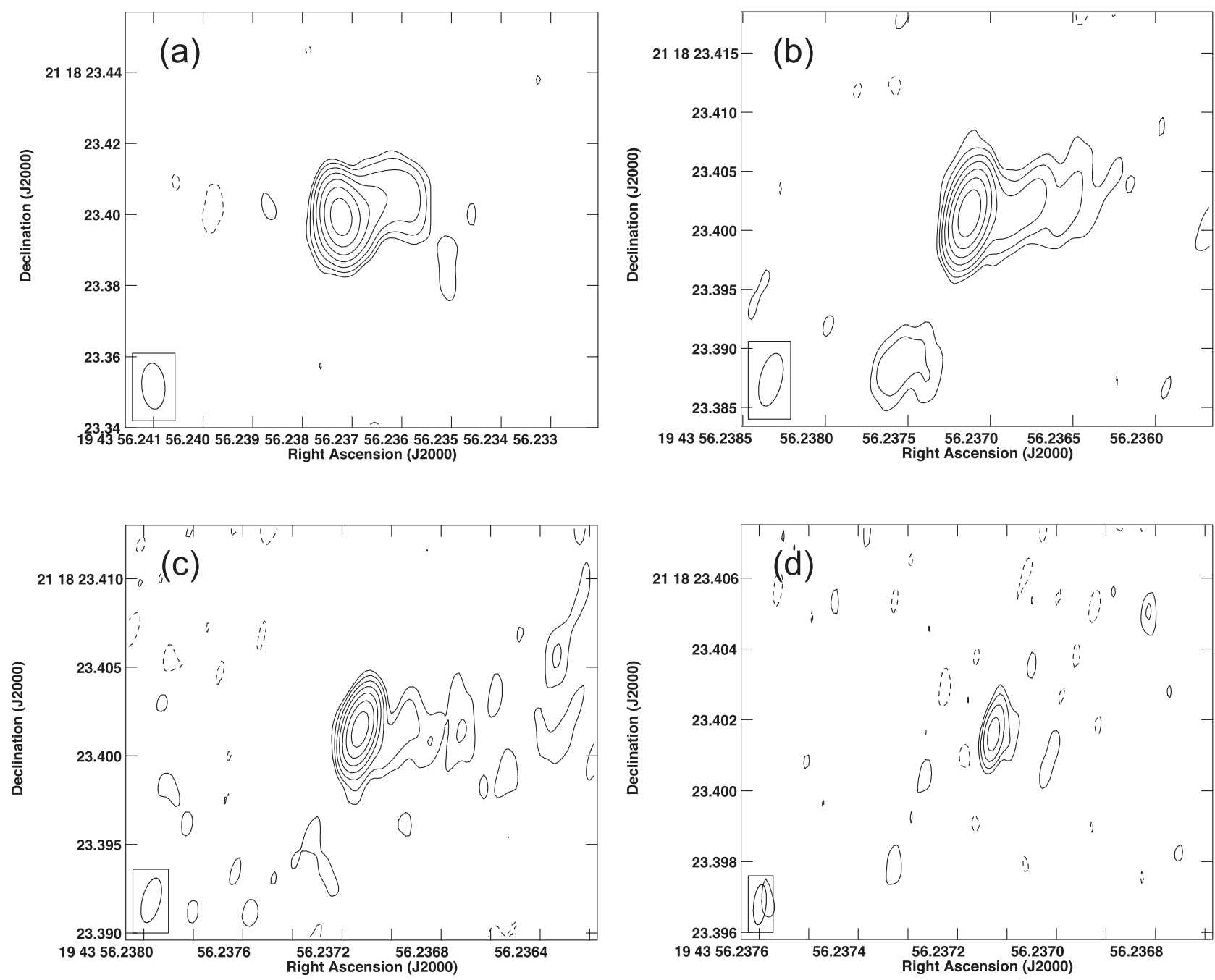

Figure 5. Contour images of HESS J1943+213 with VLBA (a) $1.6 \mathrm{GHz}$, (b) $4.3 \mathrm{GHz}$, (c) $7.6 \mathrm{GHz}$, and (d) $15 \mathrm{GHz}$ bands. Contour levels are plotted above $1 \%$ of the peak image intensity: $-1,1,2,4,8,16,32,64(1.6 \mathrm{GHz}) ;-2,-1,1,2,4,8,16,32,64$ (4.3 and $7.6 \mathrm{GHz}) ;-8,8,16,32,64(15 \mathrm{GHz})$. Negative levels are shown with dashed lines.
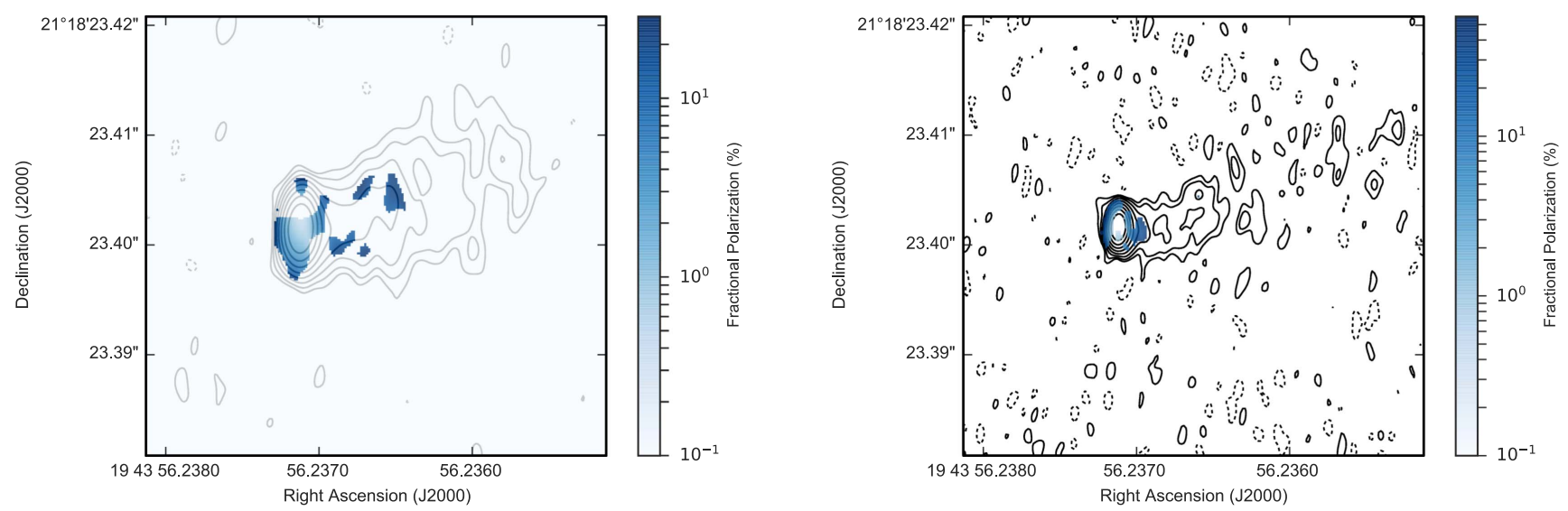

Figure 6. Epoch II VLBA $4.3 \mathrm{GHz}$ (left) and $7.6 \mathrm{GHz}$ (right) images of the HESS J1943+213 radio counterpart. The contours represent the -1 (dashed), 1, 2, 4, 8 , $16,32,64,128$ levels above $0.3 \%$ of the peak image intensity. The fractional polarization is illustrated with the color maps.

observations lacked a reference source for determining an absolute source position. Hence, we make a conservative estimate of the uncertainty in the position measurements. The uncorrected atmosphere and ionosphere will contribute $<0.1$ mas to the positional uncertainty (e.g., Hachisuka et al. 2015). The high image signal-to-noise ratio (>1000:1) 


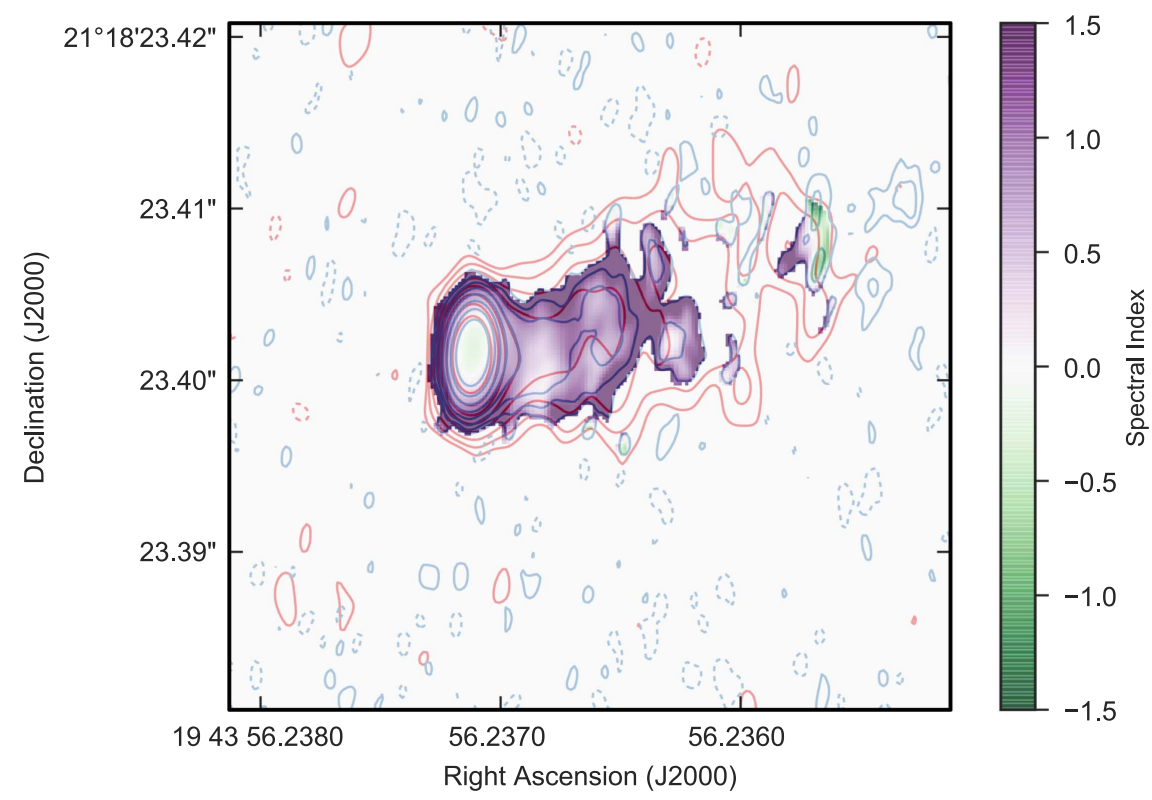

Figure 7. Spectral map of the core-jet structure of the HESS J1943+213 radio counterpart made from epoch II VLBA 4.3 and $7.6 \mathrm{GHz}$ (degraded to $4.3 \mathrm{GHz}$ resolution) band images, with contours for $4.3 \mathrm{GHz}$ (red) and $7.6 \mathrm{GHz}$ (blue) representing -1 (dotted), 1, 2, 4, 8, 16, 32, 64, 128 levels above $0.3 \%$ of the peak image intensity.

Table 3

Measurements of HESS J1943+213 Properties from Phase and Amplitude Self-calibrated VLBA Images

\begin{tabular}{|c|c|c|c|c|c|}
\hline Band & $\begin{array}{l}\text { Peak Intensity } \\
\left(\text { mJy beam }^{-1}\right)\end{array}$ & $\begin{array}{l}\text { Image Noise } \\
\left(\mathrm{mJy} \mathrm{beam}^{-1}\right)\end{array}$ & $\begin{array}{l}\text { Spectral Flux Density } \\
(\mathrm{mJy})\end{array}$ & $\begin{array}{c}\text { Core Major/Minor Axis }{ }^{\mathrm{a}} \\
\text { (mas) }\end{array}$ & $\begin{array}{l}T_{\mathrm{B}} \\
(\mathrm{K})\end{array}$ \\
\hline \multicolumn{6}{|l|}{2015} \\
\hline $4.3 \mathrm{GHz}$ & 15.252 & 0.065 & $16.25 \pm 0.12$ & $0.68 / 0.24$ & $>2.2 \times 10^{9}$ \\
\hline $7.6 \mathrm{GHz}$ & 15.032 & 0.071 & $16.23 \pm 0.13$ & $0.41 / 0.30$ & $>1.7 \times 10^{9}$ \\
\hline${ }^{\mathrm{b}} 15 \mathrm{GHz}$ & 8.1059 & 0.16 & $10.51 \pm 0.32$ & $\mathrm{~N} / \mathrm{A} / \mathrm{N} / \mathrm{A}$ & $\mathrm{N} / \mathrm{A}$ \\
\hline $4.3 \mathrm{GHz}$ & 18.388 & 0.020 & $17.12 \pm 0.047$ & $0.85 / 0.71$ & $>2.2 \times 10^{9}$ \\
\hline $7.6 \mathrm{GHz}$ & 14.877 & 0.025 & $20.39 \pm 0.041$ & $0.57 / 0.47$ & $>1.5 \times 10^{9}$ \\
\hline
\end{tabular}

Notes.

${ }^{\text {a }}$ Sizes are reported after deconvolving by the beam size.

${ }^{\mathrm{b}}$ Values are from phase-only self-calibrated images.

ensures that the statistical contribution to the uncertainty is well below $0.01 \mathrm{mas}$ and is effectively negligible. The biggest source of uncertainty is the effect of source structure on the measurements. Source structure can complicate position measurement (1) through unmatched $u v$ coverage of the two epochs, leading to slight differences in reconstruction of the source, and (2) through evolution of the source itself. The maximum contribution from source structure to the uncertainty in the position measurement can be estimated from the ratio of the core brightness to the local structure (in $\mathrm{Jy} \mathrm{beam}^{-1}$ ). At worst, this ratio is $30: 1$ and the uncertainty from source structure is $1 / 30$ of a beam width $(3 \times 1.5$ mas $)$, i.e., $\sim 0.1$ mas. Thus, a conservative estimate of 0.1 mas is adopted as the uncertainty in the position measurements between the two VLBA observations.

The source position in the $4.3 \mathrm{GHz}$ images is measured by fitting a Gaussian function to the core. The differences in the position measurements are $0.08 \pm 0.04$ mas, where the uncertainty is from the fit statistics alone. The measured difference in the position between the two epochs is smaller than the uncertainty in the position determination. We again set an upper limit on the transverse velocity. In this case, with the same source distance assumption of $17 \mathrm{kpc}$, the upper limit on the transverse velocity is $8 \mathrm{~km} \mathrm{~s}^{-1}$.

The upper limits on the transverse velocity, especially the $8 \mathrm{~km} \mathrm{~s}^{-1}$ limit from the two epochs of VLBA observations, are much lower than typical Galactic pulsar velocities-between 100 and $300 \mathrm{~km} \mathrm{~s}^{-1}$ —obtained from VLBI proper-motion measurements (Brisken et al. 2003). These velocity limits break the assumption that the source is located within the Galaxy and effectively rule out a Galactic origin for the source.

\section{Results and Discussion}

Recent publications have strongly argued that HESS J1943 +213 is a blazar, and results presented above, especially the VLBA measurements, firmly support and solidify this scenario. Based on the location of the synchrotron peak, HESS J1943 


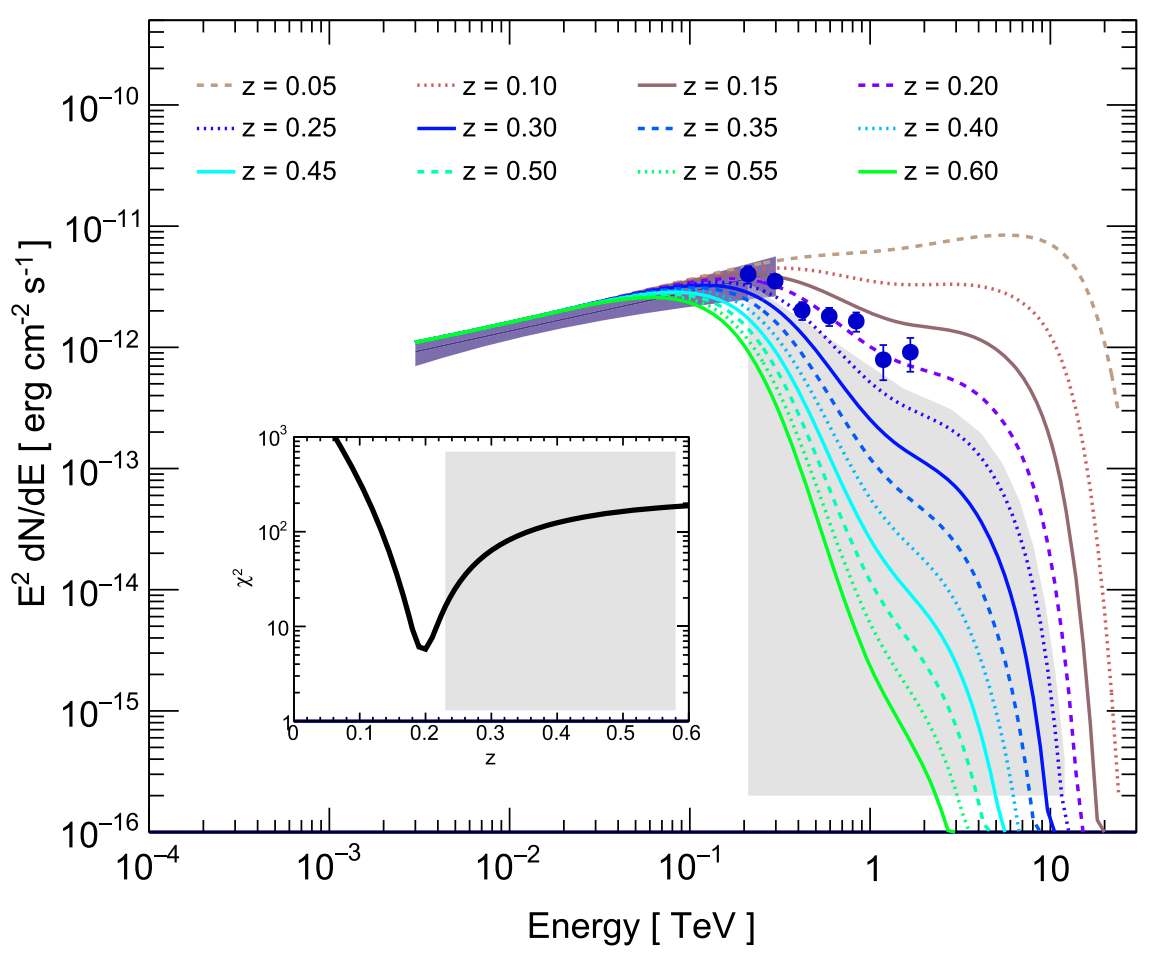

Figure 8. VERITAS observed spectrum (blue circles) fit to upper bound of the Fermi-LAT spectrum absorbed by EBL for redshift values ranging from 0 to 0.6 . The inset shows the $\chi^{2}$ distribution with redshift of the VERITAS spectrum fit to the EBL-absorbed extrapolations of the Fermi-LAT upper bound. The gray shaded areas show the $95 \%$ rejection regions.

+213 is characterized as an EHBL, a blazar subclass with very few detected members. HE $\gamma$-ray blazars behind the Galactic plane have been previously identified with Fermi-LAT (Kara et al. 2012), but HESS J1943+213 is the first such blazar also seen in VHE $\gamma$-rays.

\subsection{Redshift Constraints from Gamma-ray Spectra}

The improved detection of the inverse Compton peak with Fermi-LAT and VERITAS and the resulting higher-statistics spectra are used to set more robust upper limits on the redshift of the source. We use the same procedure for redshift estimation of HESS J1943+213 as Peter et al. (2014). In this method, the Fermi-LAT power-law spectrum is assumed to be the proxy for the intrinsic $\gamma$-ray spectrum, and downward deviations from a power-law shape are attributed to absorption effects from pair-production interactions between $\gamma$-rays and EBL photons.

Assuming a model of the EBL by Franceschini et al. (2008), the $68 \%$ upper bound of the Fermi-LAT spectrum is extrapolated into the VHE regime and absorbed for a range of redshift values. The upper bound of the Fermi-LAT spectrum is used in order for the upper limit on the redshift to be conservative. The $\chi^{2}$ value is calculated from the extrapolated Fermi-LAT upper bound and the VERITAS spectral points for each redshift value. The observed VERITAS spectrum and the extrapolated Fermi-LAT upper bounds for a range of redshifts are shown in Figure 8. The figure also includes the resulting $\chi^{2}$ distribution, which shows a minimum $\chi^{2}$ value at $z \sim 0.20$. The 95\% upper limit on the redshift derived from the $\chi^{2}$ distribution is $z<0.23$, which is significantly more constraining than the existing $z<0.45$ 95\% upper limit from Peter et al. (2014).

\subsection{Search for Flux Variability in X-Rays and $\gamma$-rays}

So far, the only claim of variability from HESS J1943+213 and its identified multiwavelength counterparts comes from measurements of different flux densities of the radio core on milliarcsecond scales using VLBI observations (Akiyama et al. 2016).

Light curves from VERITAS and Swift-XRT are presented in Figure 2 and the inset of Figure 3. A simple $\chi^{2}$ fit of a constant line to each light curve is used to test for flux variability. In addition, the fractional rms variability amplitude (Edelson et al. 1990; Rodríguez-Pascual et al. 1997) is calculated for each light curve, defined as $F_{\mathrm{var}}=\sqrt{\frac{\sigma^{2}-\delta^{2}}{\langle f\rangle^{2}}}$, where $\sigma^{2}$ is the variance of the fluxes, $\delta^{2}$ is the mean square uncertainty of the fluxes, and $\langle f\rangle$ is the mean flux. The uncertainty in $F_{\text {var }}$ is given by Equation (B2) in Vaughan et al. (2003).

The long-term VERITAS light curve is stable, with $F_{\text {var }}=0.23 \pm 0.37$ and $\chi^{2} / \mathrm{NDF}=15.6 / 12$, corresponding to a $p$-value of 0.21 for a constant flux. There is no statistically significant evidence for variability in the Swift-XRT light curve composed of three observations with $F_{\text {var }}=0.007 \pm 0.003$ and $\chi^{2} / \mathrm{NDF}=12.0 / 2$, corresponding to a $p$-value of 0.003 . In addition, there is no evidence of variability within individual XRT observations. HESS J1943+213 remains one of the most stable VHE-detected blazars.

\subsection{Modeling the HESS J1943+213 SED}

\subsubsection{SED Construction and Assumptions}

The time-averaged broadband SED of HESS J1943+213 is displayed in Figure 9. The SED is assembled using data analyzed in this work from VERITAS, Fermi-LAT, SwiftXRT, FLWO 48", and VLBA and archival SED points in the hard X-rays from the Swift Burst Alert Telescope (BAT) 


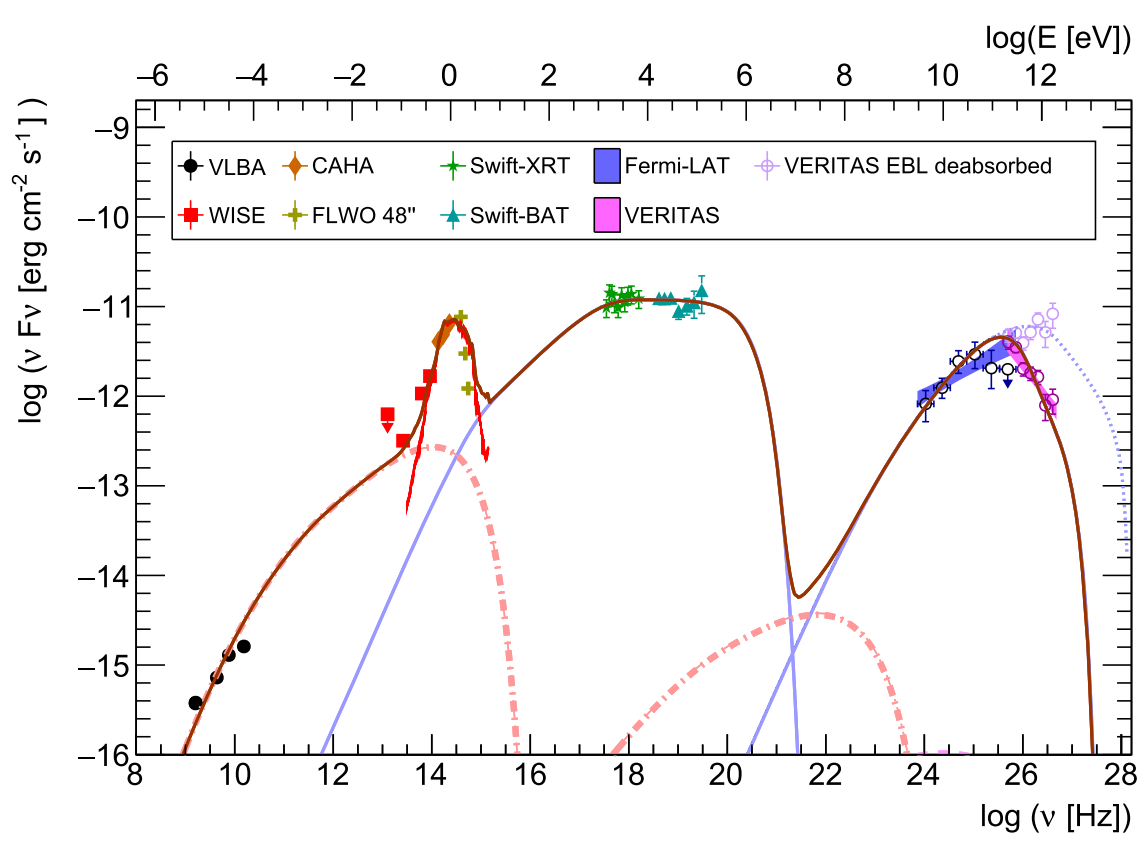

Figure 9. SED of HESS J1943+213, including the SSC model with components for a blob of relativistic particles (solid light-blue curves) and a larger jet (dot-dashed red curves). The model for the host galaxy emission is shown in solid red, while the brown curve gives the summed emission from all model components. The flux points include data from VLA $1.4 \mathrm{GHz}$, VLBA 1.6, 4.3, 7.6, $15 \mathrm{GHz}$, WISE, CAHA $3.5 \mathrm{~m}$ (Peter et al. 2014), FLWO 48", Swift-XRT and Swift-BAT, Fermi-LAT, and VERITAS. The assumed source redshift is $z=0.16$. The EBL model from Franceschini et al. (2008) is used to deabsorb the VERITAS points.

70-month survey (Baumgartner et al. 2013) and in the infrared from the Wide-field Infrared Survey Explorer (Wright et al. 2010) all-sky survey and Calar Alto Astronomical Observatory (CAHA) $3.5 \mathrm{~m}$ Telescope observations (Peter et al. 2014). As noted in previous publications (Abramowski et al. 2011; Peter et al. 2014; Tanaka et al. 2014), there is no detected cutoff in hard X-rays up to an energy of $195 \mathrm{keV}$, supporting the classification of the source as an EHBL. With the improved HE and VHE spectra of the source from VERITAS and 8 yr FermiLAT observations, respectively, the inverse Compton peak of the SED is well constrained.

For the purposes of modeling the HESS J1943+213 SED, we assume a source redshift of $z=0.16$. This redshift value is derived by repeating the redshift estimation procedure in Section 3.1 with the nominal Fermi-LAT spectrum instead of the Fermi-LAT upper bound and selecting the redshift where the $\chi^{2}$ distribution reaches a minimum. The estimate assumes that the Fermi-LAT spectrum does not deviate from a powerlaw function; hence, it is likely to be an overestimate if any downward curvature is present in the intrinsic source spectrum.

\subsubsection{Model Description and Constraints}

We model the HESS J1943+213 SED with a two-zone SSC model described by a homogeneous, compact blob within a conical wider jet. The model is a two-flow representation in which there is a highly Doppler-boosted inner jet region (blob) embedded in a wider, conical structure with a lower Lorentz factor (jet) (Hervet et al. 2015, based on Katarzyński et al. 2001). This type of model is supported by theoretical jet approaches (e.g., Sol et al. 1989), jet production mechanisms (Blandford \& Znajek 1977; Blandford \& Payne 1982), jet launching simulations (Ferreira et al. 2006), and radio VLBI imaging (Mertens \& Lobanov 2016). Our model is similar to the two-zone models used by Ghisellini et al. (2005) and Shukla et al. (2016), but with notable distinctions. The Ghisellini et al. (2005) model used a spine-layer structure, with two cylindrical components consisting of a faster-moving, higher Lorentz factor inner spine, embedded in a slower, lower Lorentz factor outer layer. The Shukla et al. (2016) model built on the spine-layer model with the addition of another SSC component near the base of the jet to represent a harder, variable emission. Unlike these models, the Hervet et al. (2015) model used here employs a conical geometry for the wider, lower Lorentz factor emission region (jet), with a spherical, higher Lorentz factor emission region (blob) embedded within.

The blob SSC component assumes an emission region composed of relativistic particles, containing tangled magnetic fields, and moving toward Earth with a Doppler factor $\delta$. The particle population is described by a broken power-law function with indices $\alpha_{1}$ and $\alpha_{2}$ and minimum, maximum, and break energies $\left(\gamma_{\min }, \gamma_{\max }\right.$, and $\left.\gamma_{\mathrm{b}}\right)$. The size of the blob is chosen to best represent the multiwavelength SED while staying close to equipartition and within the standard range of sizes for blazar models. The EBL model from Franceschini et al. (2008) is used to calculate the attenuation of $\gamma$-rays due to pair-production interactions for the SED model.

The SED model includes a near-IR and optical emission component from the host galaxy (using a PEGASE 2 template from Fioc \& Rocca-Volmerange 1999), which is characterized as a giant elliptical galaxy with a lower limit on the mass of $2.0 \times 10^{11} M_{\odot}$. The host galaxy model fits the SED data well and is the preferred description for the near-IR SED points. A nonthermal origin for this emission is unlikely and would be impossible to accommodate within the broadband SSC model. An alternative explanation for the near-IR excess is that the emission comes from a dust torus around the central black hole. This is also unlikely, however, since strong dust torus emission is not expected for HBLs and EHBLs (e.g., Meyer et al. 2011). In addition, a bright torus would induce an external-Compton signature in $\gamma$-ray energies, which we do not observe. 
Table 4

Parameters of the SSC Models for the HESS J1943+213 Broadband SED

\begin{tabular}{|c|c|c|}
\hline$\underline{\text { Value }}$ & Parameter & Unit \\
\hline \multicolumn{3}{|c|}{ General Transformation Parameters } \\
\hline 0.16 & Redshift & 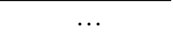 \\
\hline 71 & Hubble constant & $\mathrm{km} \mathrm{s}^{-1} \mathrm{Mpc}^{-1}$ \\
\hline 2.0 & Angle to the line of sight & $\operatorname{deg}$ \\
\hline \multicolumn{3}{|c|}{ Blob Parameters } \\
\hline 26 & Doppler factor, $\delta$ & $\ldots$ \\
\hline $3.8 \times 10^{3}$ & Particle density, $\mathrm{K}$ & $\mathrm{cm}^{-3}$ \\
\hline 1.9 & First slope of particle energy spectrum, $\alpha_{1}$ & $\cdots$ \\
\hline 3.0 & Second slope of particle energy spectrum, $\alpha_{2}$ & $\cdots$ \\
\hline $8.0 \times 10^{3}$ & Minimum electron energy Lorentz factor, & $\cdots$ \\
\hline $5.0 \times 10^{6}$ & $\begin{array}{l}\gamma_{\min } \\
\text { Maximum electron energy Lorentz factor, } \\
\gamma_{\max }\end{array}$ & $\cdots$ \\
\hline $2.0 \times 10^{5}$ & Break in electron energy spectrum, $\gamma_{\mathrm{b}}$ & $\cdots$ \\
\hline 0.1 & Magnetic field, $B$ & G \\
\hline $3.0 \times 10^{15}$ & Radius of emitting region, $R$ & $\mathrm{~cm}$ \\
\hline
\end{tabular}

Jet Parameters (First Slice)

\begin{tabular}{lll}
\hline 9 & Doppler factor & \\
$6.0 \times 10^{2}$ & Particle density & $\mathrm{cm}^{-3}$ \\
2.0 & Slope of particle energy spectrum & $\ldots$ \\
$2.0 \times 10^{2}$ & Minimum electron energy Lorentz factor & $\ldots$ \\
$1.1 \times 10^{4}$ & Maximum electron energy Lorentz factor & $\ldots$ \\
0.2 & Initial magnetic field & $\mathrm{G}$ \\
$1.2 \times 10^{16}$ & Inner radius (host galaxy frame) & $\mathrm{cm}$ \\
\hline
\end{tabular}

Jet Parameters (Global)

\begin{tabular}{llll}
\hline 8.6 & Jet length (host galaxy frame) & pc & \\
2.3 & Half-opening angle of jet (host galaxy frame) & deg & \\
50 & Number of slices & & $\ldots$ \\
0.3 & $\begin{array}{l}\text { Minimum blob-black hole distance (host } \\
\text { galaxy frame) }\end{array}$ & pc & \\
& & & \\
\hline
\end{tabular}

The VLBA and the mid-IR points are modeled with SSC emission from a stratified, conical jet. The jet model is represented as a cone discretized into 50 cylindrical slices, with increasing slice volumes at larger distances from the jet basis. Only the radius of the first slice, the jet length, and the jet opening angle are used as priors to define the jet structure. The thickness of each slice is increased logarithmically with the distance to the jet base to attain roughly the same number of particles in each slice. The radius of each slice is determined by the distance of the slice to the base and the jet opening angle. The jet speed is assumed to be constant along its propagation. The magnetic field decreases inversely with the distance to the first slice following radio measurements by O'Sullivan \& Gabuzda (2009). Jet parameters such as the magnetic field strength and particle density are provided for the first cylindrical slice in Table 4. These parameters are calculated for all other slices following an adiabatic expansion evolution. Absorption and emission coefficients are calculated for each slice.

The SSC radiation from each jet slice is used to calculate the total emission from the jet after taking into account radiation transfer through all slices in the direction of the jet propagation. The radiation transfer of the blob emission through the slices between the blob and the observer is also calculated. In addition, the external inverse Compton (EIC) interaction between the blob and the jet components is computed. The jet radiation transfer at the blob position is calculated in the jet frame. This radiation field is converted into the blob frame to calculate the EIC radiation, and the resulting EIC radiation is transferred through the jet slices in the direction of the observer. Inverse Compton radiation between the jet particles and the radiation field from the blob is assumed to be negligible. The full details of the jet model can be found in Hervet et al. (2015).

The following constraints are applied to simulate the jet emission: (1) blob velocity is greater than jet velocity, (2) the radius of the jet is larger than the radius of the blob, (3) blob $\gamma_{\max }$ is greater than jet $\gamma_{\max }$, and (4) the jet is very close to equipartition.

In addition, information from VLBA imaging of the radio counterpart presented in Section 2.5 can place additional constraints on the jet parameters. We use the size of the radio core measured from the VLBA 7.6 GHz image during Epoch 1, as it provides the most stringent constraints on jet model parameters. The jet direction appears perpendicular to the core major axis. Assuming a conical jet basis, the core major axis gives the maximum diameter of the jet basis. The projected length of the jet basis (on the sky plane) is estimated with minoraxis $-\frac{\text { majoraxis }}{2}$, which in turn gives an apparent jet half-opening angle of $65^{\circ} .2$. With the usual value of the angle between the jet axis and the line of sight of $2^{\circ}$, the intrinsic jet half-opening angle and the intrinsic radio core or jet basis length are estimated as $2^{\circ} .3$ and $8.59 \mathrm{pc}$, respectively.

\subsubsection{SED Modeling Results and Energetics Discussion}

This two-flow model is able to represent the SED very well, only slightly underestimating the lower-energy VLBA point. The $\chi^{2} / \mathrm{NDF}$ goodness-of-fit values for the blob model are $\chi_{\mathrm{XRT}}^{2} / \mathrm{NDF}=4.16 / 10, \chi_{\mathrm{BAT}}^{2} / \mathrm{NDF}=3.38 / 7, \chi_{\mathrm{LAT}}^{2} / \mathrm{NDF}=$ $3.09 / 5$, and $\chi_{\text {VERITAS }}^{2} / \mathrm{NDF}=11.33 / 7$ (note that NDF does not include the number of free parameters of the SED model). The parameters governing the wider jet model are poorly constrained, given that there is only one synchrotron slope. As such, the physical values and the energetics of this extended jet emitting zone are highly model dependent.

The full list of values of the SED model parameters can be found in Table 4 . The synchrotron peak is very broad and is located between $10^{18}$ and $10^{20} \mathrm{~Hz}$ according to the model. The minimum variability timescale predicted by the SED model is $\sim 1.24 \mathrm{hr}$ for the blob and $\sim 14.3 \mathrm{hr}$ for the jet. These timescales are not contradicted by the lack of variability detection in $\mathrm{X}$-rays and $\gamma$-rays, especially if the system is in a thermal equilibrium with constant particle injection. The large blob Doppler factor value of 26 used in the SED model leads to a low internal pair-production opacity up to the highest observed gamma-ray energies, with an optical depth of $\sim 0.03$ at $1 \mathrm{TeV}$.

The blob emission region is out of equipartition, with the energetics dominated by the kinetic energy of the particles and $u_{\mathrm{B}} / u_{e}=0.01$, where $u_{e}$ is the energy density in the particles and $u_{\mathrm{B}}$ is the energy density in the magnetic fields. The jet emission region, on the other hand, is at equipartition. The gamma-ray peak of the observed SED does not show signs of extra broadening, implying that the EIC emission is effectively negligible for the source. If the blob is too close to the central black hole, blob particles will strongly interact with synchrotron radiation from the jet basis and produce a strong EIC component. Thus, the lack of observed EIC emission can be used to place a lower limit on the distance between the blob and 
the central supermassive black hole (SMBH). Using the jet parameters of the SED model, the first jet slice is located at a distance of $0.1 \mathrm{pc}$ from the $\mathrm{SMBH}$ and the constraint on the $b l o b$-SMBH distance is $\geqslant 0.3 \mathrm{pc}$, with the gamma-ray emission peak containing $\sim 5 \%$ EIC and $95 \%$ SSC radiation.

The choice of parameters for the presented model is based on a good representation of the multiwavelength SED. Once this is achieved, an effort is made to stay close to equipartition and to reduce the total jet energetics. Parameter degeneracies, intrinsic to SSC models, cannot be fully broken by this approach. With the strong observational constraints on the synchrotron and $\gamma$-ray peaks, however, significant changes to the presented parameters will require moving away from these modeling criteria.

Given that the exact distance of the source is still uncertain, changing the assumed distance value will change the intrinsic power of the source; however, the effect on the energetics equilibria including equipartition will not be significant. A higher (lower) redshift value will yield a higher (lower) energy of the emitting particles and imply a stronger (weaker) particle acceleration mechanism. We tested models with different source redshift assumptions $(z=0.1-0.2)$ and obtained a range of likely parameters. The blob parameters with most significant changes are the Doppler factor (18-30), particle density $\left(8 \times 10^{2} \mathrm{~cm}^{-3}\right.$ to $\left.4 \times 10^{3} \mathrm{~cm}^{-3}\right)$, and radius $\left(3 \times 10^{15} \mathrm{~cm}\right.$ to $\left.7 \times 10^{15} \mathrm{~cm}\right)$.

There have been two previous efforts to model the SED of this source. Tanaka et al. (2014) modeled the IR-to- $\gamma$-ray SED using a blackbody component for the host galaxy and an SSC component. Their SSC model includes magnetic fields with a strength of $0.78 \mathrm{mG}$, a Doppler factor of 70, and a single power-law electron distribution characterized by a spectral index of $3, \gamma_{\min }=10^{5}$, and $\gamma_{\max }=3 \times 10^{7}$. The derived variability timescale in this case is $\sim 28 \mathrm{hr}$, and the model is far from equipartition with $u_{\mathrm{B}} / u_{e}=0.001$. The other SED model for the source comes from Peter et al. (2014), which represents the entire SED, including the radio regime with emission from a single population of electrons and a blackbody component for the host galaxy. In this case, the magnetic field strength is $0.05 \mathrm{G}$, the electron population is described as an exponential cutoff power-law function with $\gamma_{\min }=1, \gamma_{\max }=10^{10}$, and the energetics are out of equipartition and dominated by kinetic energy of particles with $u_{\mathrm{B}} / u_{e}=0.08$.

In terms of energy requirements, our model is able to reproduce the SED with a significantly lower value for the $\gamma_{\max }$ than in Tanaka et al. (2014) and Peter et al. (2014) and a much lower value of the Doppler factor than in Tanaka et al. (2014). The magnetic field strength in the Tanaka et al. (2014) model is lower than ours; however, this results in an emission zone that is very far from equipartition. Overall, the SED model presented in this work is able to fit the data well using more standard parameters for HBLs. In addition, the more constraining $\gamma$-ray data make our model more robust than previous attempts.

\subsection{The Role of UHECR Cascade Emission}

Despite the SSC scenario providing a good description for the HESS J1943+213 $\gamma$-ray emission, and in light of HESS $\mathrm{J} 1943+213$ being identified as an EHBL — a promising class of objects for hadronic emission-we investigate the possibility that the VHE $\gamma$-rays originate instead from electromagnetic cascades produced by interactions of UHECRs with background photon fields. To estimate the secondary $\gamma$-ray emission from such a scenario, we simulate the propagation of UHECRs
Table 5

Parameters Used in Modeling the $\gamma$-ray Data with UHECR-induced Cascade Emission

\begin{tabular}{|c|c|c|}
\hline Value & Parameter & Unit \\
\hline \multicolumn{3}{|c|}{ General Parameters } \\
\hline $124-1387$ & Source distance & $\mathrm{Mpc}$ \\
\hline 71 & Hubble constant & $\mathrm{km} \mathrm{s}^{-1} \mathrm{Mpc}^{-1}$ \\
\hline 10 & Intergalactic magnetic field strength & fG \\
\hline \multicolumn{3}{|c|}{ Cosmic-ray Parameters } \\
\hline Protons only & Composition & $\cdots$ \\
\hline 2.0 & Index of cosmic-ray spectrum & $\cdots$ \\
\hline 0.7 & Minimum cosmic-ray energy & $\mathrm{EeV}$ \\
\hline 300 & Maximum cosmic-ray energy & $\mathrm{EeV}$ \\
\hline 6.0 & Jet opening angle for cosmic rays & degrees \\
\hline
\end{tabular}

and calculate all relevant interactions using publicly available software, CRPropa3 (for details of the software package see Alves Batista et al. 2016).

Due to the uncertain distance of the source, multiple redshifts within the range defined by the lower and upper redshift limits are explored. The two parameters that largely determine the shape of the secondary $\gamma$-ray spectrum are the redshift of the source and the shape of the EBL spectrum. To represent the EBL, the model by Franceschini et al. (2008) is employed. The list of parameter values used for the simulation is provided in Table 5. The choice of intergalactic magnetic field strength, proton spectrum index, and maximum proton energy does not significantly affect the shape of the predicted secondary $\gamma$-ray spectrum but can change the total cosmic-ray power required to produce the secondary $\gamma$-rays by an order of magnitude. Following Essey \& Kusenko (2010), a Lorentz factor of 10 is assumed, corresponding to a cosmic-ray jet opening angle of $6^{\circ}$. If, instead, the cosmic-ray emission is assumed to be isotropic, the power in cosmic rays required to generate the same flux of secondary $\gamma$-rays increases by a factor of 365 .

Figure 10 illustrates the predicted secondary $\gamma$-ray spectra fit to the VERITAS data, with the condition that the VERITAS and Fermi-LAT data are not exceeded by the predicted secondary $\gamma$-ray emission. The resulting requirements on the cosmic-ray power for producing the secondary spectra are modest compared to the energy budgets of blazars (the Eddington luminosity of a typical $10^{9} M_{\odot} \mathrm{SMBH}$ powering a blazar is $10^{47} \mathrm{erg} \mathrm{s}^{-1}$ ). The shapes of the predicted secondary $\gamma$-ray spectra, however, imply that only the highest-energy end of the VERITAS spectrum-energies $>1 \mathrm{TeV}$ - can be represented with this type of emission mechanism and only for source distances closer than $z \sim 0.14$.

\section{Summary and Conclusions}

HESS $\mathrm{J} 1943+213$ is a new addition to the rare class of EHBLs with a strong detection in both HE and VHE $\gamma$-rays. The uncertainty over the source classification has been largely settled in favor of an EHBL with support from results presented here. The detection of a jet-like structure in VLBA 1.6, 4.3, and $7.6 \mathrm{GHz}$ bands and the measurements of spectral indices comparable to other known blazars for both the radio core and the jet are strong evidence in support of this position. In addition, the lack of detectable proper motion between EVN and VLBA observations and the two epochs of VLBA 


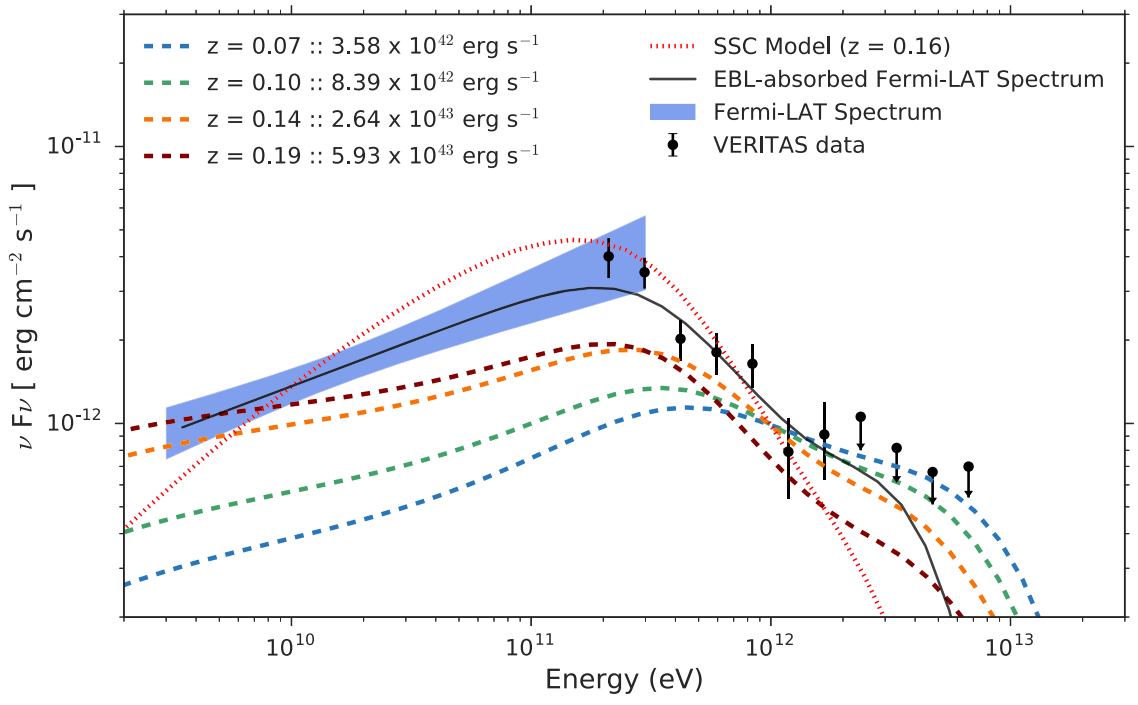

Figure 10. Predicted secondary $\gamma$-ray spectra from cascades initiated by UHECRs shown with dashed lines. The legend specifies the assumed distance and the UHECR power required to produce each spectrum. The solid black line shows the Fermi-LAT spectrum extrapolated to VHE energies and absorbed by the EBL using the model from Franceschini et al. (2008), assuming a source redshift of $z=0.16$ (near the best-fit redshift value from the redshift estimation procedure in Section 3.1). The dotted red line is the SSC model from Section 3.3.

observations set constraining upper limits on the transverse velocity as low as $8 \mathrm{~km} \mathrm{~s}^{-1}$ if the source is of Galactic origin, essentially ruling out this possibility.

Deep observations with VERITAS detect a source at the HESS J1943+213 position with $\sim 20 \sigma$ significance, producing a high-statistics spectrum. The VHE spectral properties are consistent with the measurement from H.E.S.S.; however, the VERITAS spectrum extends down to $180 \mathrm{GeV}$ energies, providing an overlap with the Fermi-LAT spectrum from 8 yr of observations. The VERITAS and the Fermi-LAT spectra together give an accurate description of the $\gamma$-ray peak of the source SED. These spectra are used to derive more stringent upper limits on the source redshift of $z<0.23$.

No statistically significant evidence of flux or spectral variability is found in data from long-term VERITAS observations, as well as in Swift-XRT observations over the course of 4 days. As EHBLs are not known for strong variability, the stability of the source is not surprising, but it is still unusual.

The improved $\gamma$-ray data are used to update and model the broadband SED of HESS J1943+213. An SSC model with a component for the infrared to optical light from the host galaxy describes the SED very well, while keeping model parameters to standard values for HBLs. The VLBA data can also be accommodated in the model with the addition of a stratified, conical jet component. Since EHBLs are candidates for hadronic emission, a possible contribution to the $\gamma$-ray portion of the SED from secondary photons produced along the line of sight by UHECR-induced cascades is explored for a range of allowed distances for the source. The shape of the secondary $\gamma$-ray spectra, however, makes them viable only for $>1 \mathrm{TeV}$ energies and only if the source is located closer than $z \sim 0.14$.

There is still much to learn from HESS J1943+213. Highsensitivity observations of HESS J1943+213 in the hard X-ray band with an instrument like NUSTAR would be valuable for characterizing both the spectral shape and the variability of the emission produced by the higher-energy particles and would help pinpoint the emission mechanism of the source. Moreover, a precise measurement of the distance to HESS J1943+213 would be of great benefit for pinning down its physical properties. With a known distance, the stability of the source, combined with its spectral properties in X-rays and $\gamma$-rays, would make it an ideal target for studies aiming to constrain the strength of intergalactic magnetic fields and to measure the density of the EBL.

This research is supported by grants from the U.S. Department of Energy Office of Science, the U.S. National Science Foundation, and the Smithsonian Institution and by NSERC in Canada. We acknowledge the excellent work of the technical support staff at the Fred Lawrence Whipple Observatory and at the collaborating institutions in the construction and operation of the instrument.

The VERITAS Collaboration is grateful to Trevor Weekes for his seminal contributions and leadership in the field of VHE $\gamma$-ray astrophysics, which made this study possible.

The VLBA is operated by the Long Baseline Observatory. The Long Baseline Observatory is a facility of the National Science Foundation operated under cooperative agreement by Associated Universities, Inc.

Facilities: VERITAS, Fermi-LAT, Swift-XRT, FLWO 48" $(1.2 \mathrm{~m})$, VLBA.

Software: Fermi Science Tools, Fermipy, HEASoft, XSpec, AIPS, CRPropa3.

\section{ORCID iDs}

W. Benbow (1) https://orcid.org/0000-0003-2098-170X

R. Bird (ib https://orcid.org/0000-0002-4596-8563

W. Cui (i) https://orcid.org/0000-0002-6324-5772

Q. Feng (i) https://orcid.org/0000-0001-6674-4238

J. P. Finley (i) https://orcid.org/0000-0002-8925-1046

A. Furniss (ㄴ) https://orcid.org/0000-0003-1614-1273

G. H. Gillanders (1) https://orcid.org/0000-0001-8763-6252

D. Hanna (1) https://orcid.org/0000-0002-8513-5603

C. A. Johnson (1) https://orcid.org/0000-0002-0641-7320

P. Kaaret (1) https://orcid.org/0000-0002-3638-0637

D. Kieda (1) https://orcid.org/0000-0003-4785-0101

M. Krause 나 https://orcid.org/0000-0001-7595-0914 
M. J. Lang (1) https://orcid.org/0000-0003-4641-4201

R. Mukherjee (1) https://orcid.org/0000-0002-3223-0754

D. Nieto (1) https://orcid.org/0000-0003-3343-0755

N. Park (1) https://orcid.org/0000-0002-4282-736X

M. Pohl (1) https://orcid.org/0000-0001-7861-1707

A. Popkow (i) https://orcid.org/0000-0002-3157-8839

E. Pueschel (ib https://orcid.org/0000-0002-0529-1973

G. T. Richards (10 https://orcid.org/0000-0002-1408-807X

K. Shahinyan (1) https://orcid.org/0000-0001-5128-4160

D. A. Williams (10) https://orcid.org/0000-0003-2740-9714

\section{References}

Abramowski, A., Acero, F., Aharonian, F., et al. 2011, A\&A, 529, A49 Acciari, V. A., Beilicke, M., Blaylock, G., et al. 2008, ApJ, 679, 1427 Acero, F., Ackermann, M., Ajello, M., et al. 2015, ApJS, 218, 23

Ackermann, M., Ajello, M., Atwood, W. B., et al. 2016, ApJS, 222, 5 Akiyama, K., Stawarz, Ł., Tanaka, Y. T., et al. 2016, arXiv:1603.00877 Alves Batista, R., Dundovic, A., Erdmann, M., et al. 2016, JCAP, 5, 038 Arnaud, K. A. 1996, in ASP Conf. Ser. 101, Astronomical Data Analysis Software and Systems V, ed. G. H. Jacoby \& J. Barnes (San Francisco, CA: ASP), 17

Baumgartner, W. H., Tueller, J., Markwardt, C. B., et al. 2013, ApJS, 207, 19 Blandford, R. D., \& Payne, D. G. 1982, MNRAS, 199, 883

Blandford, R. D., \& Znajek, R. L. 1977, MNRAS, 179, 433

Bonnoli, G., Tavecchio, F., Ghisellini, G., \& Sbarrato, T. 2015, MNRAS, 451,611

Böttcher, M. 2007, Ap\&SS, 309, 95

Brisken, W. F., Fruchter, A. S., Goss, W. M., Herrnstein, R. M., \& Thorsett, S. E. 2003, AJ, 126, 3090

Cerruti, M., Zech, A., Boisson, C., \& Inoue, S. 2015, MNRAS, 448, 910 Cogan, P. 2008, ICRC (Mérida), 30, 1385

Costamante, L., Ghisellini, G., Giommi, P., et al. 2001, A\&A, 371, 512

Daniel, M. K. 2008, ICRC (Mérida), 30, 1325

Edelson, R. A., Krolik, J. H., \& Pike, G. F. 1990, ApJ, 359, 86

Essey, W., \& Kusenko, A. 2010, APh, 33, 81

Ferreira, J., Dougados, C., \& Cabrit, S. 2006, A\&A, 453, 785

Ferrigno, C., Blasi, P., \& de Marco, D. 2004, NuPhS, 136, 191

Fioc, M., \& Rocca-Volmerange, B. 1999, arXiv:astro-ph/9912179

Fomin, V. P., Stepanian, A. A., Lamb, R. C., et al. 1994, APh, 2, 137

Fossati, G., Maraschi, L., Celotti, A., Comastri, A., \& Ghisellini, G. 1998, MNRAS, 299, 433

Franceschini, A., Rodighiero, G., \& Vaccari, M. 2008, A\&A, 487, 837

Gabányi, K. É., Dubner, G., Giacani, E., et al. 2013, ApJ, 762, 63

Ghisellini, G., \& Tavecchio, F. 2008, MNRAS, 387, 1669

Ghisellini, G., Tavecchio, F., \& Chiaberge, M. 2005, A\&A, 432, 401
Giommi, P., Padovani, P., Polenta, G., et al. 2012, MNRAS, 420, 2899

Hachisuka, K., Choi, Y. K., Reid, M. J., et al. 2015, ApJ, 800, 2

Hervet, O., Boisson, C., \& Sol, H. 2015, A\&A, 578, A69

Hillas, A. M. 1985, ICRC (San Diego, CA), 19, 445

Holder, J., Atkins, R. W., Badran, H. M., et al. 2006, APh, 25, 391

Hovatta, T., Aller, M. F., Aller, H. D., et al. 2014, AJ, 147, 143

Kalberla, P. M. W., Burton, W. B., Hartmann, D., et al. 2005, A\&A, 440, 775

Kara, E., Errando, M., Max-Moerbeck, W., et al. 2012, ApJ, 746, 159

Katarzyński, K., Sol, H., \& Kus, A. 2001, A\&A, 367, 809

Klepser, S. 2012, APh, 36, 64

Konigl, A. 1981, ApJ, 243, 700

Landi, R., Stephen, J. B., Masetti, N., et al. 2009, A\&A, 493, 893

Li, T.-P., \& Ma, Y.-Q. 1983, ApJ, 272, 317

Linford, J. D., Taylor, G. B., Romani, R. W., et al. 2012, ApJ, 744, 177

Malizia, A., Landi, R., Bassani, L., et al. 2007, ApJ, 668, 81

Marscher, A. P. 1980, ApJ, 235, 386

Mertens, F., \& Lobanov, A. P. 2016, A\&A, 587, A52

Meyer, E. T., Fossati, G., Georganopoulos, M., \& Lister, M. L. 2011, ApJ, 740, 98

Mücke, A., \& Protheroe, R. J. 2001, APh, 15, 121

Mücke, A., Protheroe, R. J., Engel, R., Rachen, J. P., \& Stanev, T. 2003, APh, 18,593

O'Sullivan, S. P., \& Gabuzda, D. C. 2009, MNRAS, 400, 26

Padovani, P., \& Giommi, P. 1995, MNRAS, 277, 1477

Park, N. \& the VERITAS Collaboration 2015, arXiv:1508.07070

Peter, D., Domainko, W., Sanchez, D. A., van der Wel, A., \& Gässler, W. 2014, A\&A, 571, A41

Reynolds, S. P. 1982, ApJ, 256, 13

Rodríguez-Pascual, P. M., Alloin, D., Clavel, J., et al. 1997, ApJS, 110, 9

Shahinyan, K. \& the VERITAS Collaboration 2015, arXiv:1508.07358

Shukla, A., Mannheim, K., Chitnis, V. R., et al. 2016, ApJ, 832, 177

Sikora, M., Begelman, M. C., \& Rees, M. J. 1994, ApJ, 421, 153

Sol, H., Pelletier, G., \& Asseo, E. 1989, MNRAS, 237, 411

Stickel, M., Fried, J. W., \& Kuehr, H. 1993, A\&AS, 98, 393

Stickel, M., Padovani, P., Urry, C. M., Fried, J. W., \& Kuehr, H. 1991, ApJ, 374, 431

Straal, S. M., Gabanyi, K. E., van Leeuwen, J., et al. 2016, arXiv:1603.01226

Tanaka, Y. T., Stawarz, Ł., Finke, J., et al. 2014, ApJ, 787, 155

Tavecchio, F., Ghisellini, G., Foschini, L., et al. 2010, MNRAS, 406, L70

The Fermi-LAT Collaboration 2017, arXiv:1702.00664

Urry, C. M., \& Padovani, P. 1995, PASP, 107, 803

van Moorsel, G., Kemball, A., \& Greisen, E. 1996, in ASP Conf. Ser. 101, Astronomical Data Analysis Software and Systems V, ed. G. H. Jacoby \& J. Barnes (San Francisco, CA: ASP), 37

Vaughan, S., Edelson, R., Warwick, R. S., \& Uttley, P. 2003, MNRAS, 345,1271

Wood, M., Caputo, R., Charles, E., et al. 2017, arXiv:1707.09551

Wright, E. L., Eisenhardt, P. R. M., Mainzer, A. K., et al. 2010, AJ, 140, 1868 